\title{
Nonlinear cointegrating power function regression with endogeneity*
}

\author{
Zhishui $\mathrm{Hu}$ \\ University of Science and Technology of China \\ Peter C. B. Phillips \\ Yale University, University of Auckland \\ University of Southampton \& Singapore Management University \\ Qiying Wang \\ The University of Sydney
}

November 18, 2020

\begin{abstract}
This paper develops an asymptotic theory for nonlinear cointegrating power function regression. The framework extends earlier work on the deterministic trend case and allows for both endogeneity and heteroskedasticity, which makes the models and inferential methods relevant to many empirical economic and financial applications, including predictive regression. A new test for linear cointegration against nonlinear departures is developed based on a simple linearized pseudo-model that is very convenient for practical implementation and has standard normal limit theory in the strictly exogenous regressor case. Accompanying the asymptotic theory of nonlinear regression, the paper establishes some new results on weak convergence to stochastic integrals that go beyond the usual semi-martingale structure and considerably extend existing limit theory, complementing other recent findings on stochastic integral asymptotics. The paper also provides a general framework for extremum estimation limit theory that encompasses stochastically nonstationary time series and should be of wide applicability.
\end{abstract}

\section{JEL Classification: C13, C22.}

Key words and phrases: Nonlinear power regression, Least squares estimation, Nonstationarity, Endogeneity, Heteroscedasticity.

${ }^{*}$ The authors thank the Co-Editor, Pentti Saikkonen, and two referees for helpful comments on the original manuscript, which have led to many improvements. Hu acknowledges research support from NSFC (No. 11671373); Wang acknowledges research support from Australian Research Council; Phillips acknowledges research support from the NSF under Grant No. 18-50860 and the Kelly Fund at the University of Auckland. Address correspondence to Qiying Wang, School of Mathematics and Statistics, The University of Sydney, NSW 2006, Australia; e-mail: qiying.wang@sydney.edu.au. 


\section{Introduction}

Since the initial work by Park and Phillips (2001) in this area, the past two decades have witnessed significant developments in nonlinear cointegrating regression, including parametric, nonparametric and semi-parametric specifications of such models. These developments have provided a framework of econometric estimation and inference for a wide class of nonlinear, nonstationary relationships. Among many other contributions to this research, we may refer to Chang, Park and Phillips (2001), Chang and Park (2003), Bae and de Jong (2007), Wang and Phillips (2009a, b, 2016), Kim and Kim (2012) and Gao and Phillips (2013), together with the references cited therein.

In recent work Chan and Wang (2015) established some general results on nonlinear parametric cointegrating regression. In comparison with previous research, Chan and Wang (2015) employed a different approach to investigating asymptotics in models of this kind. Their approach directly established joint distributional convergence of the martingale of interest in conjunction with its conditional variance, rather than relying on the classical approach to the martingale limit theorem which requires convergence in probability for the conditional variance. ${ }^{1}$ The methodology used in Chan and Wang (2015) has important advantages since it is usually difficult to prove convergence in probability without expanding the probability space, particularly in the structure of cointegrating regression settings where the conditional variance typically converges weakly to a random variable rather than in probability to a constant. The latter methodology was used in Park and Phillips (2001) and requires more restrictive conditions as well as expansion of the probability space to secure the required results.

The models considered in Chan and Wang (2015) include integrable and non-integrable regression functionals. However, as is apparent from their Assumption 3.4, power regression functions are excluded, such as those that take the form $f(x)=\beta|x|^{\gamma}$, where $\beta \in \mathbb{R}$ and $\gamma \geq 0$. This shortcoming in coverage is restrictive because power function regression is a commonly used model in many empirical applications. An area of application where such regression has been found particularly useful is in testing the validity and order of polynomial regression (Baek, Cho and Phillips, 2015; Cho and Phillips, 2018.)

One goal of the present paper is to address this omission in coverage. A further goal is to contribute to the general development of asymptotic theory in nonlinear nonstationary regression. First, while this paper focuses on power function regression, our results allow for models

\footnotetext{
${ }^{1}$ Readers are referred to Wang (2014) for a recent general exposition and development of limit theory relevant to nonstationary time series regression.
} 
that include both endogeneity and heteroskedasticity. Power functions fall within the framework of homogeneous functions that were considered in Park and Phillips (2001), but their results applied to $I(1)$ integrated and weakly exogenous regressor processes and martingale difference equation errors with constant conditional variances, thereby excluding a wide class of nonstationary processes and standard error volatility models such as ARCH and GARCH. Second, accompanying the development of our asymptotic theory for power regression, we provide new results on convergence to stochastic integrals that extend beyond the semimartingale structure. Since the 1980s there has been extensive research in both econometrics and probability on weak convergence to stochastic integrals, yielding a large body of useful theory. But results that extend beyond a semimartingale framework and allow for nonlinear functionals have only recently become available, notably by Liang, et al. (2016) and Peng and Wang (2018). However, the nonlinear functionals considered in the latter papers exclude power functions such as $f(x)=\beta|x|^{\gamma}$, since the first order derivative of $f(x)$ does not everywhere exist or even satisfy a Lipschitz condition in cases such as $-1<\gamma<0$.

The present paper contributes to this literature by building a framework of theory that accommodates these extensions, thereby helping to complete the limit theory for extremum estimation in nonlinear nonstationary regressions. To achieve this purpose the paper provides a weak convergence result for normalized stochastic processes, associated sample covariance functionals, and quadratic variations at a level of generality that assists in delivering asymptotics for power regression. Further, as in Phillips (2007) where deterministic power function regression was analyzed, we show how different convergence rates apply in corresponding least squares power regressions in the presence of stochastic trends.

The paper is organized as follows. Section 2 develops limit theory in a stochastic power function regression model. Certain technical results concerning weak convergence to stochastic integrals that extend beyond semimartingale formulations are provided in Section 3. We mention that these results are quite different from previous researches and are of some independent interest. They are designed to allow for a class of nonlinear locally integrable functions that are suited to power function regression modeling. Section 4 concludes, proofs of all the main results are given in Section 5, and Appendix A provides a framework of extremum estimation for nonlinear least squares estimation that allows for various convergence rates and asymptotic linearization with general forms of score and hessian functions that allow for many different forms of limit theory.

Throughout the paper $\Rightarrow$ denotes weak convergence of probability measures with respect to the uniform topology (see, for instance, Billingsley (1968)) and $\rightarrow_{D}$ is weak convergence in 
Euclidean space. For a symmetric matrix $A$, we take $A>0$ to mean that $A$ is positive definite. A function $g(x)$ is called locally integrable if, for all compact sets $K \subset \mathbb{R}, \int_{K}|g(x)| d x<\infty$.

\section{Nonlinear cointegrating power regression}

Let $\left(\xi_{k}, u_{k}\right)_{k \geq 1}$ be a sequence of arbitrary random vectors. Consider a nonlinear cointegrating power regression model defined by

$$
y_{k}=\beta\left|x_{k}\right|^{\gamma}+u_{k}
$$

where $x_{k}=\sum_{j=1}^{k} \xi_{j}$ and $\theta=(\beta, \gamma) \in \Theta:=\mathbb{R} \times[-1 / 2, \infty)$. The least squares estimator (LSE) $\tilde{\theta}_{n}$ of $\theta$ is defined by the extremum problem

$$
\widetilde{\theta}_{n}=\left(\widetilde{\beta}_{n}, \widetilde{\gamma}_{n}\right)=\underset{(\beta, \gamma) \in \Theta}{\arg \min } \sum_{k=1}^{n}\left(y_{k}-\beta\left|x_{k}\right|^{\gamma}\right)^{2} .
$$

To develop asymptotics for the estimator $\widetilde{\theta}_{n}$, we denote the true parameter $\theta_{0}=\left(\beta_{0}, \gamma_{0}\right)$, where $\beta_{0} \neq 0$ and $\gamma_{0}>-1 / 2$. The power parameter $\gamma$ is clearly unidentified when $\beta_{0}=0$ and only weakly identified when the true regression coefficient $\beta_{0}$ is local to zero in the sense that $\beta_{0}=o(1)$ as $n \rightarrow \infty$. The latter case fits within the weak instrument econometric literature and has been analyzed by Shi and Phillips (2012) in the nonstationary regressor case and Andrews and Cheng (2012) in the stationary case. ${ }^{2}$ In addition, when $\gamma_{0}<-1 / 2$ there are difficulties in developing asymptotics for the LSE $\widetilde{\theta}_{n}$, as discussed in Remark 2.1 below $^{3}$. Throughout the present work we maintain the assumption that $\beta_{0} \neq 0$ and $\gamma_{0}>-1 / 2$.

Write $x_{n k}=x_{k} / d_{n}$ where $d_{n}^{2}=\operatorname{var}\left(x_{n}\right)$ and $u_{n k}=\frac{1}{\sqrt{n}} \sum_{j=1}^{k} u_{j}$. Throughout the paper, we assume

$$
\frac{1}{n} \sum_{k=1}^{n} d_{k}^{-1} d_{n}=O(1), \quad d_{n} \rightarrow \infty \quad \text { and } \quad d_{n}^{2}=O\left(n^{\mu}\right)
$$

for some $0<\mu<2$. This is a minor requirement and holds for usual $I(1)$ processes and a partial sum of a long memory process. We further make use of the following conditions:

A1 (i) $\left\{u_{k}, \mathcal{F}_{k}\right\}_{k \geq 1}$ forms a martingale difference with $\sup _{k \geq 1} \mathbb{E} u_{k}^{2}<\infty$, where $\left\{\mathcal{F}_{k}\right\}_{k \geq 1}$ is a filtration such that $x_{k}$ is adapted to $\mathcal{F}_{k-1}$ for all $k \geq 1\left(\mathcal{F}_{0}\right.$ is defined to be a trivial $\sigma$-field).

\footnotetext{
${ }^{2}$ Weak identification occurs in such cases because the loading coefficient parameter $\beta$ of the nonlinear function may be close to zero and limit theory under the alternative typically fails to provide a good approximation to finite sample behavior close to the null. Development of a local limit theory that improves the approximation uniformly well irrespective of the strength of the identification relies on uniform weak convergence of sample covariance functionals to stochastic integral limits - see Shi and Phillips (2012).

${ }^{3}$ Related but less complex convergence issues arise in the deterministic (evaporating) trend case with $x_{k}=k$ for which $\sum_{k=1}^{\infty} k^{2 \gamma}<\infty$ when $\gamma<-1 / 2$ and the usual excitation condition for consistency fails.
} 
(ii) There exists a 2-dimensional continuous Gaussian process $\left(X_{t}, U_{t}\right)$ with covariance matrix $\Omega_{t}>0$ so that

$$
\left(x_{n,[n t]}, u_{n,[n t]}\right) \Rightarrow\left(X_{t}, U_{t}\right), \quad \text { on } D_{\mathbb{R}^{2}}[0,1]
$$

Condition A1 imposes a martingale structure in the model (2.1), which is extensively used in the literature of nonlinear cointegrating regression. See, for instance, Park and Phillips (2001) and Chan and Wang (2015). However, unlike these existing results, only $\sup _{k \geq 1} \mathbb{E} u_{k}^{2}<\infty$ rather than $\sup _{k \geq 1} \mathbb{E}\left(u_{k}^{2} \mid \mathcal{F}_{k-1}\right) \leq C<\infty$ is used here, which allows for heteroskedasticity in the model (2.1), thereby enhancing wider use of our results in financial econometrics. The martingale difference error structure in model (2.1) will be extended to include endogeneity in Section 2.1 and more general models are considered in Section 2.2.

Let $F_{n}=\operatorname{diag}\left[\sqrt{n} d_{n}^{\gamma_{0}} / \log d_{n}, \sqrt{n} d_{n}^{\gamma_{0}}\right]$ be a diagonal rate matrix, $Q_{n}(\theta)=\sum_{k=1}^{n}\left(y_{k}-\right.$ $\left.\beta\left|x_{k}\right|^{\gamma}\right)^{2}$ and $S_{n}(\theta)=\frac{\partial Q_{n}(\theta)}{\partial \theta}$. Our first result concerning the asymptotic behavior of the extremum estimator $\widetilde{\theta}_{n}$ follows. The theorem is stated as a local rather than a global result because the objective function (2.2) is not a convex function of the parameters unless more restrictive conditions on $\gamma$ are introduced, which makes a general proof of consistency challenging. Although it is unclear whether the sequence $\widetilde{\theta}_{n}$ defined by $(2.2)$ is the same as the sequence of roots $\hat{\theta}_{n}$ in the theorem below, they must coincide with probability approaching to 1 if the minimization $(2.2)$ is taken over a set shrinking at an appropriate rate ${ }^{4}$.

Theorem 2.1. Suppose A1 holds. For any $\gamma_{0}>0$, there exists a sequence of estimators $\hat{\theta}_{n}$ such that $S_{n}\left(\hat{\theta}_{n}\right)=0$ with probability approaching to 1 , and

$$
F_{n}\left(\hat{\theta}_{n}-\theta_{0}\right) \rightarrow_{D} \quad\left(\begin{array}{c}
1 \\
-1 / \beta_{0}
\end{array}\right) \frac{V_{0} \int_{0}^{1}\left|X_{t}\right|^{\gamma_{0}} \log \left|X_{t}\right| d U_{t}-V_{1} \int_{0}^{1}\left|X_{t}\right|^{\gamma_{0}} d U_{t}}{V_{1}^{2}-V_{0} V_{2}}
$$

where $V_{i}=\int_{0}^{1}\left|X_{t}\right|^{2 \gamma_{0}} \log ^{i}\left|X_{t}\right| d t$ for $i=0,1$ and 2 .

Since $X_{t}$ has a continuous path, for $\gamma_{0} \geq 0$ the existence of $V_{i}$ and the limit distribution on the right hand of (2.4) follow immediately. Further. when the Gaussian limit processes $\left(X_{t}, U_{t}\right)$ are independent, the limit distribution in (2.4) is mixed normal. Notably, the limit distribution is degenerate, reflecting the intimate linkage between the roles of the coefficient and power parameters in $\theta=(\beta, \gamma)$ and the associated singularity of the limiting distribution arising from the asymptotic collinearity of the induced regressors in the linearized system corresponding to the model (2.1). This phenomenon mirrors the singular limit distribution behavior that was

\footnotetext{
${ }^{4}$ Indeed, as noted by one of the referees, if $F_{n}\left(\widetilde{\theta}_{n}-\theta_{0}\right)=O_{P}(1)$ then Theorem 2.1 and (2.4) give the limit distribution of $\widetilde{\theta}_{n}$.
} 
analyzed in Phillips (2007) in the context of power function deterministic trend regressors. In particular, the asymptotic collinearity in deterministic trend regressors such as $|t|^{\gamma}$ and $|t|^{\gamma} \log (t)$ also arises with stochastic trend regressors such as $\left|x_{t}\right|^{\gamma}$ and $\left|x_{t}\right|^{\gamma} \log \left|x_{t}\right|$. These variables arise in a natural way in Taylor expansions of the first order conditions in the nonlinear power function regressions that are explored in this paper. The asymptotic collinearity leads to a degenerate limit distribution for the estimated parameters, as occurs in Theorem 2.1. The problem was discussed in detail in Phillips (2007) to which readers may refer for further details.

Figs. 1(a) and 1(b) show kernel density estimates for the estimates $\widehat{\beta}_{n}$ and $\widehat{\gamma}_{n}$ for sample sizes $n \in\{100,250,500,750\}$ computed for $\beta=1$ and $\gamma=0.5$ from 50,000 replications. The simulation DGP used a random walk regressor $x_{t}$ with iid $\mathcal{N}(0,1)$ innovations and independent $u_{t} \sim$ iid $\mathcal{N}(0,1)$ equation errors. Both estimates show increasing concentration as $n$ increases and greater kurtosis than Gaussianity in line with the corresponding mixed normal limit distribution of Theorem 2.1 that applies in this exogenous regressor case. Evidently, the densities of $\widehat{\gamma}_{n}$ given in Fig. 1(b) display much smaller variation than those of $\widehat{\beta}_{n}$, consonant with the greater rate of convergence of the power parameter estimate $\widehat{\gamma}_{n}$. In spite of their different variation, these finite sample distributions show the same general shape characteristics, reflecting the singularity of the limit distribution established in Theorem 2.1.

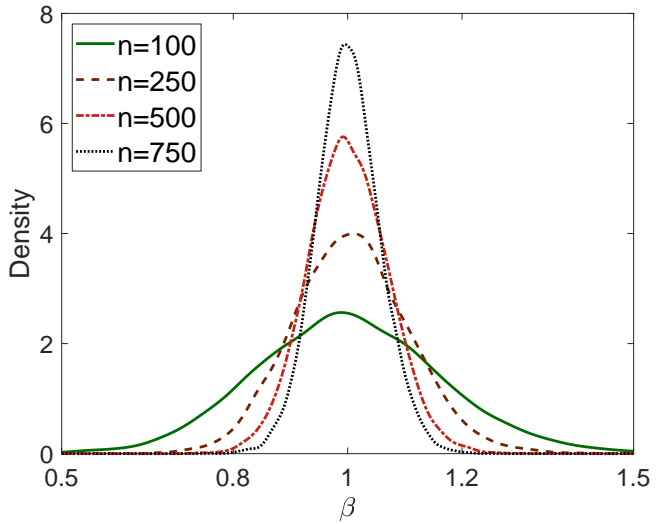

(a) $\widehat{\beta}_{n}$ densities

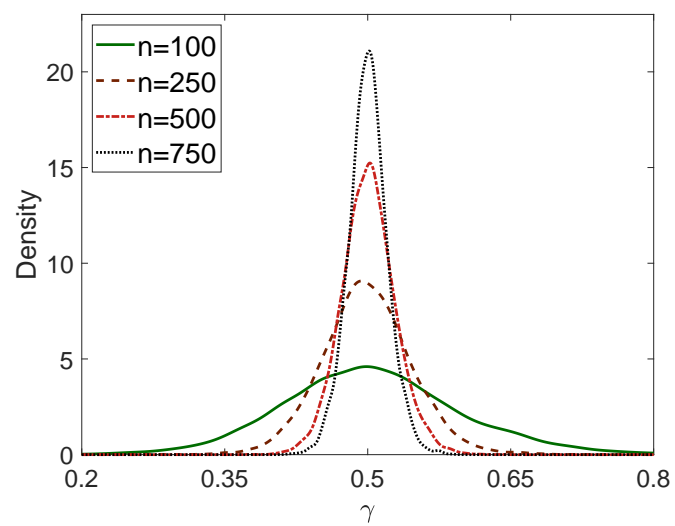

(b) $\hat{\gamma}_{n}$ densities

Figure 1: Empirical densities of the estimates $\widehat{\beta}_{n}$ and $\widehat{\gamma}_{n}$ for sample sizes $n \in\{100,250,500,750\}$ with $\beta=1$ and $\gamma=0.5$.

Theorem 2.1 can be extended to include the extra domain $-1 / 2<\gamma_{0} \leq 0$ of the power parameter under the following additional condition:

A2 (i) $x_{k} / d_{k}$ has a density $p_{k}(x)$ that is uniformly bounded by a constant $K$ for all $1 \leq k \leq n$ and $x \in \mathbb{R}$, 
(ii) $X_{t}$ has a density $\widetilde{p}_{t}(x)$ satisfying $\sup _{x} \int_{0}^{1} \widetilde{p}_{t}(x) d t<\infty$,

(iii) $\sup _{k \geq 1} \mathbb{E}\left(u_{k}^{2} \mid \mathcal{F}_{k-1}\right) \leq C<\infty$.

We mention that $\mathbf{A 2}$ (i) and (ii) ensure the existence of $V_{i}$ for $-1 / 2<\gamma_{0} \leq 0$ and the smoothness condition on the density of $x_{k} / d_{k}$ is needed for the convergence of the sample quantities to $V_{i}$. See, for instance, Pötscher (2004) and Berkes and Horváth (2006). Further discussion is given in Section 3. Since the limit result involves locally integrable functionals of integrated processes, we also require the more restrictive $\mathbf{A 2}$ (iii) instead of $\sup _{k \geq 1} \mathbb{E} u_{k}^{2}<\infty$ for technical reasons. This condition can be modified under higher moment conditions on $u_{k}$ and a narrower interval of validity for the power parameter $\gamma_{0}$. These extensions involve some complex further calculations and are therefore not pursued in the present work.

Theorem 2.2. Suppose $\boldsymbol{A} 1$ and $\boldsymbol{A} 2$ hold. The limit theory (2.4) continues to hold for $-1 / 2<$ $\gamma_{0} \leq 0$.

Remark 2.1. If $\gamma_{0}<-1 / 2$, the random variable $V_{2}$ does not exist even in the case where the process $X_{t}$ is standard Brownian motion $U_{t}$ because the integral fails to converge. Note, in particular, that $\int_{0}^{1}\left|U_{t}\right|^{2 \gamma_{0}} d t=\infty$ a.s. when $\gamma_{0}<-1 / 2$. See, e.g., Ethier and Kurtz (1986, p. 332). In consequence, use of the present methods fail and the asymptotic behavior of $\hat{\theta}_{n}$ is unclear in such cases and a matter for further investigation.

Remark 2.2. The proof of (2.4) heavily depends on the existence of the integral $\int_{0}^{1}\left|X_{t}\right|^{2 \gamma_{0}-\epsilon} d t$ for some small $\epsilon>0$ rather than just only on the existence of $V_{i}$ itself. Since $|x|^{-\epsilon}$ even for small $\epsilon>0$ is locally Lebesque rather than Riemann integrable on $\mathbb{R}, \mathbf{A 2}$ (i) and (ii) are essentially required to ensure the existence of $\int_{0}^{1}\left|X_{t}\right|^{-\epsilon} d t$, as seen in Theorems 1.2 and 2.1 of Berkes and Horváth (2006). This helps to explain why Theorem 2.1 excludes the case $\gamma_{0}=0$, but this can be established under the additional condition A2, as seen in Theorem 2.2.

Remark 2.3. If $\gamma>-1 / 2$ is fixed and known, the least squares estimator (LSE) $\hat{\beta}(\gamma)$ of $\beta$ in model (2.1) is given by

$$
\hat{\beta}(\gamma)=\frac{\sum_{k=1}^{n} y_{k}\left|x_{k}\right|^{\gamma}}{\sum_{k=1}^{n}\left|x_{k}\right|^{2 \gamma}}=\beta_{0}+\frac{\sum_{k=1}^{n}\left|x_{k}\right|^{\gamma} u_{k}}{\sum_{k=1}^{n}\left|x_{k}\right|^{2 \gamma}} .
$$

Under the given conditions (A2 is required if $\gamma<0$ ), it is readily seen that

$$
\sqrt{n} d_{n}^{\gamma}\left[\hat{\beta}(\gamma)-\beta_{0}\right] \rightarrow_{D} \frac{\int_{0}^{1}\left|X_{t}\right|^{\gamma} d U_{t}}{\int_{0}^{1}\left|X_{t}\right|^{2 \gamma} d t}
$$

In comparison with (2.4), there is now a different convergence rate for the asymptotic distribution of $\hat{\beta}(\gamma)$. This phenomenon was noted by Phillips (2007) in the investigation of nonlinear power trend regression. 
Remark 2.4. Using (2.4), we have

$$
\sqrt{n} d_{n}^{\gamma_{0}}\left(\widehat{\gamma}_{n}-\gamma_{0}\right) \rightarrow_{D} \frac{1}{\beta_{0}} \frac{V_{1} \int_{0}^{1}\left|X_{t}\right|^{\gamma_{0}} d U_{t}-V_{0} \int_{0}^{1}\left|X_{t}\right|^{\gamma_{0}} \log \left|X_{t}\right| d U_{t}}{V_{1}^{2}-V_{0} V_{2}},
$$

where $V_{i}=\int_{0}^{1}\left|X_{t}\right|^{2 \gamma_{0}} \log ^{i}\left|X_{t}\right| d t$ for $i=0,1$ and 2 , as before. Since $\beta_{0}$ is consistently estimable, this limit theory enables model specification of linear cointegration, which involves testing the null hypothesis ${ }^{5}$

$$
\mathcal{H}_{0}: \gamma=1 \quad \text { vs } \quad \mathcal{H}_{1}: \gamma \neq 1
$$

Indeed a test statistic that may be used to test (2.8) can be defined by

$$
T_{n}=\sqrt{n} \widehat{d}_{n} \widehat{\beta}(1)\left(\widehat{\gamma}_{n}-1\right)
$$

where $\widehat{\beta}(1)$ is given as in Remark 2.3 and $\widehat{d}_{n}$ is constructed so that $\widehat{d}_{n} / d_{n} \rightarrow 1$ in probability. From (2.6) and (2.7) it follows that under the null $\mathcal{H}_{0}$,

$$
T_{n} \rightarrow_{D} \frac{\widetilde{V}_{1} \int_{0}^{1}\left|X_{t}\right| d U_{t}-\widetilde{V}_{0} \int_{0}^{1}\left|X_{t}\right| \log \left|X_{t}\right| d U_{t}}{\widetilde{V}_{1}^{2}-\widetilde{V}_{0} \widetilde{V}_{2}},
$$

where $\widetilde{V}_{i}=\int_{0}^{1}\left|X_{t}\right|^{2} \log ^{i}\left|X_{t}\right| d t$ for $i=0,1$ and 2. Under the conditions that allow for the joint distribution of $\left(X_{t}, U_{t}\right)$ to be determined, this test is asymptotically pivotal and consistent with $P_{\mathcal{H}_{1}}\left(\left|T_{n}\right| \geq t_{0}\right) \rightarrow 1$ for any $t_{0}>0$, where $P_{\mathcal{H}_{1}}(\cdot)$ denotes the probability under the alternative $\mathcal{H}_{1}$. In more general situation where the joint distribution of $\left(X_{t}, U_{t}\right)$ depends on some unknown parameters $(\vartheta$, say $), T_{n}$ is not pivotal. In this case, a bootstrap or other simulation method will be required for inference. Furthermore, some aspects of inference, such as confidence interval construction, are more difficult. The limit distribution of $\hat{\theta}_{n}$ given in (2.4) depends jointly on the parameter vector $\theta_{0}=\left(\beta_{0}, \gamma_{0}\right)$, making direct inference about $\theta_{0}$ in power regression more complex. It is not clear at present whether or not an asymptotic theory might be developed for $\hat{\theta}_{n}$ using a different approach such as a self-normalized quantity in place of the use of rate matrix scaling like $F_{n}$ in (2.4) so that the unknown parameter $\theta_{0}$ on the right hand of (2.4) can be eliminated and an asymptotically pivotal approach developed.

Remark 2.5. A more convenient approach to testing $\mathcal{H}_{0}$ that avoids nonlinear regression is to fit a linear regression based on a first order Taylor approximation (or pseudo-linear version) of

\footnotetext{
${ }^{5} \mathrm{~A}$ related approach to testing the linear cointegrating specification based on the hypothesis (2.8) is to use the augmented regressor approach of Baek et al. (2015), where a quasi-likelihood ratio statistic is developed and identification problems associated with models with power transform regressors are discussed for stationary and trend stationary models. In the present setting, the method involves estimating the augmented cointegrating regression $y_{k}=\beta\left|x_{k}\right|+\alpha\left|x_{k}\right|^{\gamma}+u_{k}$, and testing the significance of the nonlinear component via the parameter $\alpha$. Nonlinear estimation of the power coefficient $\gamma$ is again required with this approach.
} 
the original model (2.1), viz., $y_{k}=\beta\left|x_{k}\right|^{\gamma}+u_{k}$, about the same model with coefficient $\beta_{0}$ and power coefficient $\gamma_{0}=1$. This (infeasible) pseudo-linear model has the explicit linear regression form

$$
\tilde{y}_{k}\left(\beta_{0}\right)=\beta\left|x_{k}\right|+\gamma z_{k}\left(\beta_{0}\right)+u_{k}=q_{k}\left(\beta_{0}\right)^{\prime} \theta+u_{k}, \quad z_{k}\left(\beta_{0}\right)=\beta_{0}\left|x_{k}\right| \log \left(\left|x_{k}\right|\right),
$$

where $\tilde{y}_{k}\left(\beta_{0}\right)=y_{k}+\beta_{0}\left|x_{k}\right| \log \left(\left|x_{k}\right|\right)$ and $q_{k}\left(\beta_{0}\right)=\left(\left|x_{k}\right|, z_{k}\left(\beta_{0}\right)\right)^{\prime}$. The model (2.11) is linear in both the coefficients $(\beta, \gamma)$ and the variables $\left(\left|x_{k}\right|, z_{k}\left(\beta_{0}\right)\right)$, given $\beta_{0}$. The unknown parameter $\beta_{0}$ in the variables $\tilde{y}_{k}\left(\beta_{0}\right)$ and $z_{k}\left(\beta_{0}\right)$ in $(2.11)$ can be estimated consistently under the null $\mathcal{H}_{0}:$ $\gamma=1$ using the linear cointegrating regression estimate $\hat{\beta}(1)=\left(\sum_{k=1}^{n}\left|x_{k}\right|^{2}\right)^{-1}\left(\sum_{k=1}^{n}\left|x_{k}\right| y_{k}\right)$ in place of the unknown $\beta_{0}$. The resulting feasible regression equation is then fitted by linear least squares of $\tilde{y}_{k}(\hat{\beta}(1))$ on $q_{k}(\hat{\beta}(1))$. The null hypothesis $\mathcal{H}_{0}: \gamma=1$ is tested by applying a standard $t$-test of $\gamma=1$ in this fitted linear regression, which we write as

$$
\tilde{y}_{k}(\hat{\beta}(1))=\hat{\beta}\left|x_{k}\right|+\hat{\gamma} z_{k}(\hat{\beta}(1))+\hat{u}_{k}=q_{k}(\hat{\beta}(1))^{\prime} \hat{\theta}+\hat{u}_{k}
$$

The required $t$-statistic associated with $\hat{\gamma}$ in this regression has the usual form $t_{\gamma}=\frac{\hat{\gamma}-1}{s_{\gamma}}$ with $s_{\gamma}^{2}=s_{u}^{2} / m_{\gamma \gamma}, s_{u}^{2}=n^{-1} \sum_{k=1}^{n} \hat{u}_{k}^{2}, \hat{u}_{k}$ defined by the regression residual in $(2.12)$, and $m_{\gamma \gamma}$ the second diagonal element of $M_{\theta \theta}^{-1}$, where

$$
\begin{aligned}
& M_{\theta \theta}=\sum_{k=1}^{n} q_{k}(\hat{\beta}(1)) q_{k}(\hat{\beta}(1))^{\prime}=\left(\begin{array}{cc}
\sum_{k=1}^{n}\left|x_{k}\right|^{2} & \hat{\beta}(1) \sum_{k=1}^{n}\left|x_{k}\right|^{2} \log \left|x_{k}\right| \\
\hat{\beta}(1) \sum_{k=1}^{n}\left|x_{k}\right|^{2} \log \left|x_{k}\right| & \hat{\beta}(1)^{2} \sum_{k=1}^{n}\left|x_{k}\right|^{2} \log ^{2}\left|x_{k}\right|
\end{array}\right) \\
& =:\left(\begin{array}{cc}
\Omega_{n 0} & \hat{\beta}(1) \Omega_{n 1} \\
\hat{\beta}(1) \Omega_{n 1} & \hat{\beta}(1)^{2} \Omega_{n 2}
\end{array}\right) .
\end{aligned}
$$

Then, $\hat{\theta}-\theta=M_{\theta \theta}^{-1} \sum_{k=1}^{n} q_{k}(\hat{\beta}(1))^{\prime} u_{k}$ which under the linear cointegration null $\mathcal{H}_{0}: \gamma=1$ gives

$$
\hat{\gamma}-1=\frac{1}{\hat{\beta}(1)\left(\Omega_{n 2} \Omega_{n 0}-\Omega_{n 1}^{2}\right)} \sum_{k=1}^{n}\left(\Omega_{n 0}\left|x_{k}\right| \log \left|x_{k}\right|-\Omega_{n 1}\left|x_{k}\right|\right) u_{k}
$$

with $t$-statistic

$$
t_{\gamma}=m_{\gamma \gamma}^{1 / 2}(\hat{\gamma}-1) / s_{u}=\frac{1}{s_{u} \Omega_{n 0}^{1 / 2}\left(\Omega_{n 2} \Omega_{n 0}-\Omega_{n 1}^{2}\right)^{1 / 2}} \sum_{k=1}^{n}\left(\Omega_{n 0}\left|x_{k}\right| \log \left|x_{k}\right|-\Omega_{n 1}\left|x_{k}\right|\right) u_{k} .
$$

When $\left(x_{n,[n t]}, u_{n,[n t]}\right) \Rightarrow\left(X_{t}, U_{t}\right)$ where $\left(u_{k}, \mathcal{F}_{k}\right)$ is a martingale difference sequence so that $x_{k}$ is adapted to $\mathcal{F}_{k-1}$, we deduce, just as in result (2.4), that

$$
t_{\gamma} \rightarrow_{D} \frac{\Omega_{0} \int_{0}^{1}\left|X_{t}\right| \log \left|X_{t}\right| d U_{t}-\Omega_{1} \int_{0}^{1}\left|X_{t}\right| d U_{t}}{\sigma \Omega_{0}\left(\Omega_{2} \Omega_{0}-\Omega_{1}^{2}\right)^{1 / 2}}
$$

where $\Omega_{j}=\int_{0}^{1}\left|X_{t}\right|^{2} \log ^{j}\left|X_{t}\right| d t$. If the limit processes $\left(X_{t}, U_{t}\right)$ are independent, it follows that $t_{\gamma} \rightarrow_{D} N(0,1)$, giving a simple test of linear cointegration. When $\left(X_{t}, U_{t}\right)$ are dependent, it seems that some form of fully modified regression statistic is required for pivotal inference, as in the nonlinear regression case based on Theorem 2.1. 


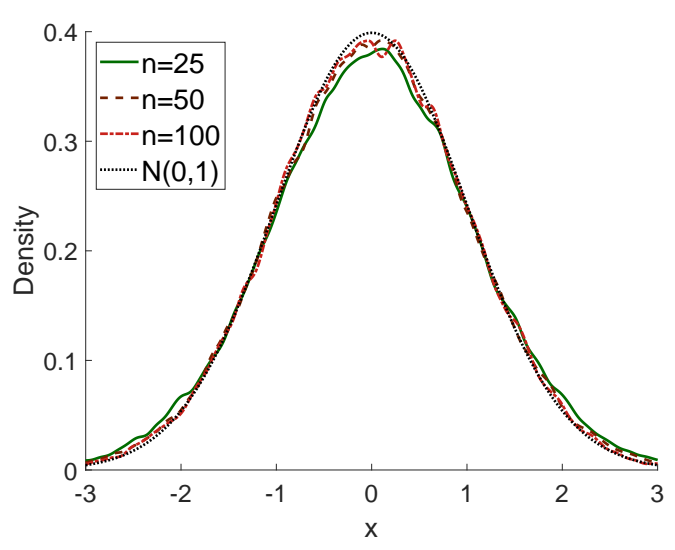

(a) $t_{\gamma}$ densities under $\mathcal{H}_{0}: \gamma=1$

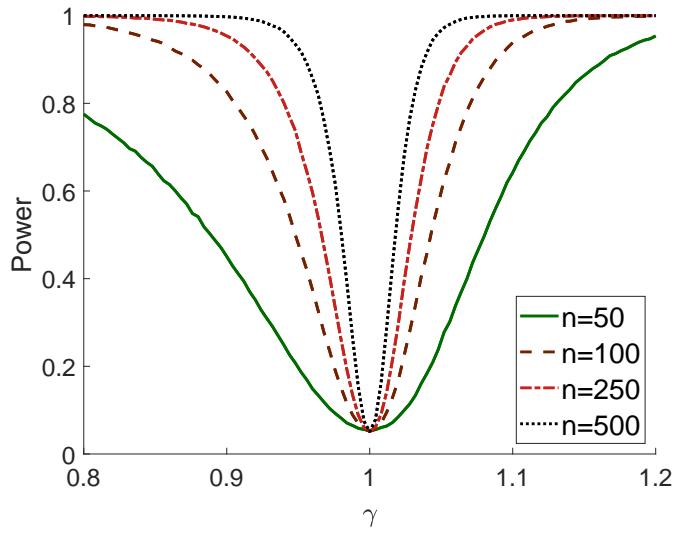

(b) Power curves of $t_{\gamma}$

Figure 2: Empirical densities of the $t$-statistic $t_{\gamma}=\frac{\hat{\gamma}-1}{s_{\gamma}}$ in the pseudo-linear model regression (2.12) under $\mathcal{H}_{0}: \gamma=1$ and power curves for $\gamma \in[0.8,1.2]$ for various sample sizes $n$, based on 50,000 replications and a model with random walk $x_{t}$ and $i i d \mathcal{N}(0,1)$ errors.

Fig. 2(a) displays kernel density estimates of the finite sample densities of the statistic $t_{\gamma}$ under the null $\gamma=1$ and with $\beta_{0}=1.0$ for sample sizes $n=25,50,100$ shown against the standard normal density $\mathcal{N}(0,1)$. The same data generating mechanism as the earlier simulation was used, with random walk $x_{t}$ and iid $\mathcal{N}(0,1)$ errors. Evidently, the finite sample distributions are all well approximated by the standard normal even for small $n$ in this case. Fig. 2(b) gives power function curves of the test based on a nominal asymptotic $5 \%$ significance level for a two tailed test, for a grid of values $\gamma \in[0.8,1.20]$ and sample sizes $n \in\{100,200,300,500\}$. Test size is close to the nominal $5 \%$ level, conforming with the graphs of the densities of $t_{\gamma}$ under the null $\gamma=1$. The power curves show that power rises monotonically for deviations of the power coefficient $\gamma$ from unity in all cases and for both $\gamma<1$ and $\gamma>1$. There is some evidence of slightly stronger local power against alternative with $\gamma>1$ rather than $\gamma<1$, which may arise from the amplified effect of the regressor $\left|x_{k}\right|^{\gamma}$ in the generating model $y_{k}=\beta\left|x_{k}\right|^{\gamma}+u_{k}$ for the data $y_{k}$ in this case. Overall, the findings indicate that this simple $t$-test of $\mathcal{H}_{0}: \gamma=1$ has good size and power for testing linear cointegrating regression in the exogenous regressor case and is easy to implement in practical work.

Remark 2.6. In a natural setting amenable to a linear cointegrated structure, it may be desirable to consider the following nonlinear power function cointegrating regression model

$$
y_{k}=\alpha^{\prime} z_{k}+\beta\left|x_{k}\right|^{\gamma}+u_{k}
$$

where $\alpha=\left(\alpha_{1}, \ldots, \alpha_{d}\right)^{\prime}, \beta \in \mathbb{R}$, and $-1 / 2<\gamma<1$ are unknown parameters, $z_{k}$ is a $d$-dimensional regressor whose differences $\Delta z_{k}=z_{k}-z_{k-1}$ are stationary, and $x_{k}$ and $u_{k}$ are defined as in 
Theorem 2.1. In applications related to cointegration analysis and forecasting based on usual linear regression formulations, the power term $\beta\left|x_{k}\right|^{\gamma}$ in model (2.13) may provide an extra precision correction term that admits nonlinear effects that are relevant in certain empirical examples. As discussed above, the presence of a power regressor term in such cointegrating regression is a mechanism for testing linearity, as was done in Baek et al. (2015) and Cho and Phillips (2018) in stationary and deterministic trend model settings. The limit theory in the present paper provides a foundation for a general study of such formulations and tests, in addition to the approach based on the test $T_{n}$ given in (2.9). Full investigation of this topic in the present context requires challenging new limit theorems, which are deferred to later work.

\subsection{Extension to endogeneity}

The data generating process in model (2.1) is assumed to have a martingale structure. This assumption is used in many articles in parametric cointegrating regression and predictive regression. See, for instance, Chang, Park and Phillips (2001), Park and Phillips (2001), Phillips (2015) and Chan and Wang (2015). From the viewpoint of empirical applications, however, this martingale structure can be restrictive. The aim of this section is to remove the restriction so that endogeneity is allowed in the model. Explicitly, we are concerned with the model:

$$
y_{k}=\beta\left|x_{k}\right|^{\gamma}+w_{k}
$$

where $x_{k}$ is the partial sum process $x_{k}=\sum_{j=1}^{k} \xi_{j}$,

$$
w_{k}=u_{k}+z_{k-1}-z_{k}
$$

the $u_{k}$ are assumed to satisfy $\mathbf{A} \mathbf{1}$, and the $z_{k}$ satisfy certain regularity conditions that are specified as follows:

$$
\begin{aligned}
& \text { A3 (i) } \sup _{k \geq 1} \mathbb{E}\left[\left(1+\left|z_{k-1}\right|\right)\left(1+\left|\xi_{k}\right|\right)\right]^{\alpha}<\infty \text { for some } \alpha>1 ; \\
& \text { (ii) } \mathbb{E} z_{k-1} \xi_{k} \rightarrow A_{0} \text {, as } k \rightarrow \infty ; \\
& \text { (iii) } \sup _{k \geq 2 m}\left|\mathbb{E}\left(\lambda_{k} \mid \mathcal{F}_{k-m}\right)\right|=o_{P}(1) \text {, as } m \rightarrow \infty \text {, where } \lambda_{k}=z_{k-1} \xi_{k}-\mathbb{E} z_{k-1} \xi_{k} \text {. }
\end{aligned}
$$

The process $\left\{w_{k}\right\}_{k \geq 1}$ in (2.15) was used by Peng and Wang (2018) in an investigation of weak convergence to stochastic integrals beyond the usual semimartingale structure. The extension arises because $\left\{w_{k}\right\}_{k \geq 1}$ is usually not a martingale difference, but the partial sum process $\sum_{k=1}^{n} w_{k}=\sum_{k=1}^{n} v_{k}+z_{0}-z_{n}$ provides an approximation to a martingale, just as in the decomposition of Phillips and Solo (1992). Such martingale approximations have been widely studied in the literature. As shown in Peng and Wang (2018), A3 introduces endogeneity by virtue of A3(ii) and allows for the following processes that are relevant in much time series econometric work: 
(i) $\xi_{k}$ is a long memory process and $w_{k}$ is a stationary causal process such as time series generated by TAR and bilinear models;

(ii) both $\xi_{k}$ and $w_{k}$ are stationary causal processes; and

(iii) $\left(\xi_{k}, w_{k}\right)_{k \geq 1}$ is near-epoch dependent, particularly a sequence of $\alpha$-mixing random variables.

Within this framework, we can estimate $\theta$ and develop asymptotic theory for the estimator $\hat{\theta}_{n}$ of $\theta$ under model (2.14). We set $F_{n}=\operatorname{diag}\left[\sqrt{n} d_{n}^{\gamma_{0}} / \log d_{n}, \sqrt{n} d_{n}^{\gamma_{0}}\right]$ as earlier, as have the following result. As in Theorem 2.1, let $Q_{n}(\theta)=\sum_{k=1}^{n}\left(y_{k}-\beta\left|x_{k}\right|^{\gamma}\right)^{2}$ and $S_{n}(\theta)=\frac{\partial Q_{n}(\theta)}{\partial \theta}$.

Theorem 2.3. Suppose that $d_{n}^{2} / n \rightarrow \infty, \boldsymbol{A} \mathbf{1}$ and $\boldsymbol{A} 3$ hold. For any $\gamma_{0}>0$, there exists a sequence of estimators $\hat{\theta}_{n}$ such that $S_{n}\left(\hat{\theta}_{n}\right)=0$ with probability approaching to 1 , and

$$
F_{n}\left(\hat{\theta}_{n}-\theta_{0}\right) \rightarrow_{D}\left(\begin{array}{c}
1 \\
-1 / \beta_{0}
\end{array}\right) \frac{V_{0} \int_{0}^{1}\left|X_{t}\right|^{\gamma_{0}} \log \left|X_{t}\right| d U_{t}-V_{1} \int_{0}^{1}\left|X_{t}\right|^{\gamma_{0}} d U_{t}}{V_{1}^{2}-V_{0} V_{2}}
$$

where, for $i=0,1,2, V_{i}$ are defined as in Theorem 2.1. If $\boldsymbol{A} 2$ holds in addition, we still have (2.16) for any $1 / \alpha<\gamma_{0} \leq 1$.

The condition that $d_{n}^{2} / n \rightarrow \infty$ is satisfied if $\xi_{k}$ is a long memory process, in which case we usually have $d_{n}^{2}=\operatorname{var}\left(\sum_{k=1}^{n} \xi_{k}\right)=n^{\mu}$ for some $1 \leq \mu<2$. See, Wang et al. (2003), for instance. Due to the fast convergence rate involving $d_{n}$ in $F_{n}$, the additional term involving $z_{k}$ in the equation error (2.15) does not produce a bias term in the limit distribution. But elimination of the bias term requires a more restrictive condition on the interval in which the real parameter $\gamma_{0}$ is located. More explanation can be found in Remark 2.7 given discussed below.

The situation is different if $d_{n}^{2} / n \rightarrow \sigma^{2}<\infty$, which generally holds if $x_{k}$ is a partial sum of a short memory process $\xi_{k}$. In this case, as the following theorem shows, the additional term $z_{k}$ has an essential impact on the limit distribution. Explicitly, when $0<\sigma<\infty$, the additional term $z_{k}$ contributes a bias term in comparison with (2.16). It is interesting to notice that, when $\sigma=0$ (i.e., $d_{n} / \sqrt{n} \rightarrow 0$ ), the additional term $z_{k}$ dominates and the convergence rate of $\hat{\theta}_{n}-\theta_{0}$ becomes slow. It seems that this phenomenon was unnoticed in previous research even in the case of linear cointegrating regression.

Theorem 2.4. Suppose that $d_{n} / \sqrt{n} \rightarrow \sigma$ with $0 \leq \sigma<\infty, \boldsymbol{A} \mathbf{1}$ and $\boldsymbol{A} 3$ hold. For any $\gamma_{0}>0$, there exists a sequence of estimators $\hat{\theta}_{n}$ such that $S_{n}\left(\hat{\theta}_{n}\right)=0$ with probability approaching to 1 , and

$$
\frac{d_{n}}{\sqrt{n}} F_{n}\left(\hat{\theta}_{n}-\theta_{0}\right) \rightarrow_{D} \quad\left(\begin{array}{c}
1 \\
-1 / \beta_{0}
\end{array}\right) \frac{V_{0} W_{1}-V_{1} W_{0}}{V_{1}^{2}-V_{0} V_{2}}
$$


where, for $i=0,1,2, V_{i}$ are defined as in Theorem 2.1 and

$$
W_{i}=\sigma \int_{0}^{1}\left|X_{t}\right|^{\gamma_{0}} \log ^{i}\left|X_{t}\right| d U_{t}+A_{0} \int_{0}^{1}\left|X_{t}\right|^{\gamma_{0}-1}\left(\gamma_{0} \log ^{i}\left|X_{t}\right|+i\right) \operatorname{sign}\left(X_{t}\right) d t
$$

for $i=1,2$. If in addition that $\boldsymbol{A} 2$ holds, we still have (2.17) for any $1 / \alpha<\gamma_{0} \leq 1$.

Remark 2.7. As explained in Remark 2.1, to ensure the existence of the functionals $W_{i}$, some restriction on the range of the real parameter $\gamma_{0}$ is essentially necessary, in the present case amounting to the condition $\gamma_{0}>0$ because of the presence of the factor $\left|X_{t}\right|^{\gamma_{0}-1}$ in the integrand of the second component of $W_{i}$. Moreover, there is a trade off between the condition $\gamma_{0}>1 / \alpha$ used in Theorem 2.4 and the moment condition on $z_{k}$ as is apparent from the condition assumed in A3 (i). It is not clear at the moment whether or not the moment condition on $z_{k}$ can be improved without restricting the interval where $\gamma_{0}$ lies.

\section{$2.2 \quad$ Further extension}

Phillips (2007) considered a regression model in the following form:

$$
y_{k}=\alpha+\beta l(k)+u_{k},
$$

where $l(x)$ is a function slowly varying at $\infty$. When $l(x)=\log x,(2.18)$ becomes the semilogarithmic growth model, which raises naturally in the study of growth convergence problems and economic transition. Since the sample moment matrix of the regressors is asymptotically singular, model (2.18) fails to fit within the usual framework. Phillips (2007) investigated asymptotics of $\operatorname{LSE}(\hat{\alpha}, \hat{\beta})$ of $(\alpha, \beta)$ by using a second order approximation of $l(x n)$ by $l(n)$ for any $x \in \mathbb{R}$.

Using similar arguments as in Phillips (2007) and the results developed in Section 3, model (2.18) can be extended to a stochastic slowly evolving trend model defined as follows

$$
y_{k}=\alpha+\beta l\left(\left|x_{k}\right|\right)+u_{k},
$$

where $x_{k}=\sum_{j=1}^{k} \xi_{j}$ and the $u_{k}$ are assumed to satisfy A1. Let $\left(\alpha_{0}, \beta_{0}\right)$ be the true parameter of $(\alpha, \beta)$. We have the following theorem.

Theorem 2.5. Suppose $\boldsymbol{A} 1$ and $\boldsymbol{A} 2$ hold and $l(x)$ satisfies the following condition: there exists $\epsilon(\lambda) \rightarrow 0$ as $\lambda \rightarrow \infty$ such that

$$
\int_{-A}^{A}\left|\frac{l(|x| \lambda)}{l(\lambda)}-1-\epsilon(\lambda) \log \right| x|| d x=o[\epsilon(\lambda)],
$$

for any fixed $A>0$. For the $L S E(\hat{\alpha}, \hat{\beta})$ of $(\alpha, \beta)$, we have

$$
F_{1 n}\left[\left(\begin{array}{c}
\hat{\alpha}_{n} \\
\hat{\beta}_{n}
\end{array}\right)-\left(\begin{array}{c}
\alpha_{0} \\
\beta_{0}
\end{array}\right)\right] \rightarrow_{D}\left(\begin{array}{c}
1 \\
-1
\end{array}\right) \frac{\int_{0}^{1} \log \left(\left|X_{t}\right|\right) d U_{t}-U_{1} \int_{0}^{1} \log \left(\left|X_{t}\right|\right) d t}{\int_{0}^{1} \log ^{2}\left(\left|X_{t}\right|\right) d t-\left[\int_{0}^{1} \log \left(\left|X_{t}\right|\right) d t\right]^{2}},
$$

where $F_{1 n}=\operatorname{diag}\left\{\sqrt{n} l\left(d_{n}\right) \epsilon\left(d_{n}\right), \sqrt{n} \epsilon\left(d_{n}\right)\right\}$. 
We remark that condition (2.20) is weak and is satisfied by the majority of slowly varying functions. Typical examples include $l(x)=\log ^{k} x, k=1,2, \ldots$, etc. For details, see Phillips (2007). The martingale structure given in A1 is essential for the establishment of Theorem 2.5. Indeed, in the proof of Theorem 2.5, we need to handle sample covariances of the type $S_{n}=\sum_{k=1}^{n} \log \left|x_{n k}\right| u_{k}$. Since $d \log x / d x=1 / x$ is not locally integrable, as seen in Section 3 , we cannot provide asymptotics for $S_{n}$ in the case where $u_{k}$ is replaced by $w_{k}$. This was also noticed in de Jong (2002). If $l(x)$ satisfies certain continuity conditions rather than being slowly varying at $\infty$, it is possible to modify model (2.19) so that endogeneity is allowed. For details, we refer to Peng and Wang (2018).

\section{Convergence to stochastic integrals: beyond semimartingale structures}

We maintain the same notation as in Section 2, except when explicitly mentioned. Technical results concerning joint weak convergence to stochastic integrals are fundamental in both linear and nonlinear nonstationary limit theory where sample covariances typically converge to stochastic integrals with respect to the relevant limiting stochastic processes rather than to normal distributions via central limit theory. The results given here extend previous research on this topic and should be helpful in future lines of research that require joint weak convergence to stochastic integrals under very general conditions. In particular, our results allow for a class of nonlinear locally integrable functions that accommodate power function regressions.

Our first result provides a framework of joint weak convergence to stochastic integrals that accommodates the normalized process, sample moments of nonlinear functions and sample covariances. This result, which follows in a long tradition of similar results, delivers the technical tools needed to establish the main limit Theorems $2.1-2.4$ given in Section 2 because of its allowance for locally integrable functions and hence power functions in regression models.

Theorem 3.1. Suppose $\boldsymbol{A} 1$ holds. For any continuous functions $g(s)$ and $f(s)$, we have

$$
\begin{aligned}
& \left(x_{n,[n t]}, u_{n,[n t]}, \frac{1}{n} \sum_{k=1}^{n} g\left(x_{n k}\right), \frac{1}{\sqrt{n}} \sum_{k=1}^{n-1} f\left(x_{n k}\right) u_{k}\right) \\
\Rightarrow & \left(X_{t}, U_{t}, \quad \int_{0}^{1} g\left(X_{t}\right) d t, \quad \int_{0}^{1} f\left(X_{t}\right) d U_{t}\right),
\end{aligned}
$$

on $D_{\mathbb{R}^{4}}[0,1]$. If $\boldsymbol{A} \mathcal{2}$ holds in addition, we still have (3.1) whenever $g(x)$ and $f^{2}(x)$ are locally integrable.

Aspects of the first part of Theorem 3.1 are known in the existing literature. See, for instance, Kurtz and Protter (1991) and Hansen (1992). Extension to locally integrable functions seems to 
be new and in such cases the condition A2 is essentially necessary to ensure the existence of the stochastic integrals in the limit. Applying Theorem 3.1 to the functions $g(x)=|x|^{2 \gamma} \log ^{m_{1}}|x|$ and $f(x)=|x|^{\gamma} \log ^{m_{2}}|x|$, where $m_{1}, m_{2} \geq 0$ are integers, we have the following corollary, which plays a key role in the proofs of the main results in the paper.

Corollary 3.1. Suppose $\boldsymbol{A} 1$ holds. For all $\gamma>0$, we have

$$
\begin{aligned}
W_{n}(\gamma) & :=\left(\frac{1}{n} \sum_{k=1}^{n}\left|x_{n k}\right|^{2 \gamma} \log ^{m_{1}}\left|x_{n k}\right|, \frac{1}{\sqrt{n}} \sum_{k=1}^{n}\left|x_{n k}\right|^{\gamma} \log ^{m_{2}}\left|x_{n k}\right| u_{k}\right) \\
& \rightarrow_{D}\left(\int_{0}^{1}\left|X_{t}\right|^{2 \gamma} \log ^{m_{1}}\left|X_{t}\right| d t, \int_{0}^{1}\left|X_{t}\right|^{\gamma} \log ^{m_{2}}\left|X_{t}\right| d U_{t}\right),
\end{aligned}
$$

jointly for all integers $m_{1}, m_{2} \geq 0$. If $\boldsymbol{A} \mathscr{2}$ holds in addition, we still have (3.2) for $-1 / 2<\gamma \leq 0$.

Let $C[a, \infty)$ denote the set of all continuous real-valued functions defined on the interval $[a, \infty)$ endowed with the topology of uniform convergence on compacta (see, for instance, van der Vaart and Wellner (1996), Ch. 1.6). With an index $\gamma$ that satisfies $\gamma \geq a>-1 / 2$, it is readily seen that $\left\{W_{n}(\gamma), n \geq 1\right\}$ is a sequence of random processes defined on the space $C[a, \infty)$. Consequently, we may extend Corollary 3.1 to the following form of functional convergence for the process $W_{n}(\gamma)$.

Theorem 3.2. Suppose $\boldsymbol{A} 1$ holds. On $C[a, \infty)$ with $a>0$, for any integers $m_{1}, m_{2} \geq 0$ we have

$$
W_{n}(\gamma) \Rightarrow\left(\int_{0}^{1}\left|X_{t}\right|^{2 \gamma} \log ^{m_{1}}\left|X_{t}\right| d t, \int_{0}^{1}\left|X_{t}\right|^{\gamma} \log ^{m_{2}}\left|X_{t}\right| d U_{t}\right)
$$

If $\boldsymbol{A} 2$ holds in addition, we still have (3.3) on $C[a, \infty)$ with $a>-1 / 2$.

In related work to Theorem 3.1 and Corollary 3.1 on convergence to stochastic integrals that sought generality beyond a semimartingale structure, Liang, et al. (2016) and Wang (2015, Section 4.5) obtained weak convergence results of sample quantities such as $\sum_{k=0}^{n-1} f\left(x_{n k}\right) w_{k}$, where $w_{k}=\sum_{j=0}^{\infty} \varphi_{j} u_{k-j}$, with $\varphi=\sum_{j=0}^{\infty} \varphi_{j} \neq 0$ and $\sum_{j=0}^{\infty} j\left|\varphi_{j}\right|<\infty$, and $u_{k}$ defined as in A1(i). More recently, Peng and Wang (2018) provided another result on such sample covariances by using the error process representation $w_{k}=u_{k}+z_{k-1}-z_{k}$ given in (2.15) instead of the martingale difference $u_{k}$. While these results are useful, the functions $f(x)$ that are employed satisfy strong smoothness conditions that require $f^{\prime}(x)$ to be continuous and satisfy a Lipschitz condition. Such conditions are clearly not satisfied for functions that arise in power regression of the form $f(x)=|x|^{\gamma} \log ^{k}|x|$, where $k \geq 0$ are integers, particularly, in the case where $f(x)$ is locally integrable (i.e., $\gamma<0$ ). 
The aim of the following theorems is to fill this gap, providing new results on convergence to stochastic integrals for the purpose of this paper. We mention that these extensions are non-trivial. To resolve the limit theory, we need to use methodology involving truncation and continuous functional approximation to a locally integrable function. We mention here that the ideas developed in the proofs seem promising for use in even more general situations such as convex functions, although those extensions are not pursued in the present work.

For use in the following, recall that $w_{k}=u_{k}+z_{k-1}-z_{k}$, as defined in (2.15).

Theorem 3.3. Suppose that $d_{n}^{2} / n \rightarrow \infty$ and $\boldsymbol{A} \mathbf{1}$ and $\boldsymbol{A} 3$ hold. Then, for any $\gamma>1$, any integer $m \geq 0$, and any continuous function $g(s)$, we have

$$
\begin{aligned}
& \left(x_{n,[n t]}, u_{n,[n t]}, \frac{1}{n} \sum_{k=1}^{n} g\left(x_{n k}\right), \frac{1}{\sqrt{n}} \sum_{k=1}^{n-1}\left|x_{n k}\right|^{\gamma} \log ^{m}\left|x_{n k}\right| w_{k}\right) \\
\Rightarrow & \left(X_{t}, U_{t}, \quad \int_{0}^{1} g\left(X_{t}\right) d t, \quad \int_{0}^{1}\left|X_{t}\right|^{\gamma} \log ^{m}\left|X_{t}\right| d U_{t}\right),
\end{aligned}
$$

on $D_{\mathbb{R}^{4}}[0,1]$. If $\boldsymbol{A} \mathscr{2}$ holds in addition, then (3.4) remains valid for any $1 / \alpha<\gamma \leq 1$, any integer $m \geq 0$, and any locally integrable function $g(x)$, where $\alpha$ is given in $\boldsymbol{A} 3(i)$.

As noted in Section 2, the rate condition $d_{n}^{2} / n \rightarrow \infty$ usually holds if $\xi_{k}$ is a long memory process. The result is different if $\xi_{k}$ is a short memory process or equivalently $d_{n}^{2} / n \rightarrow \sigma^{2}<\infty$ $(\sigma=0$ is allowed $)$, as seen in the following theorem.

Theorem 3.4. Suppose that $d_{n} / \sqrt{n} \rightarrow \sigma$ with $0 \leq \sigma<\infty$, and $\boldsymbol{A} 1$ and $\boldsymbol{A} 3$ hold. For any $\gamma>1$, any integer $m \geq 0$ and any continuous function $g(s)$, we have

$$
\begin{aligned}
& \left(x_{n,[n t]}, u_{n,[n t]}, \frac{1}{n} \sum_{k=1}^{n} g\left(x_{n k}\right), \frac{d_{n}}{n} \sum_{k=1}^{n-1}\left|x_{n k}\right|^{\gamma} \log ^{m}\left|x_{n k}\right| w_{k}\right) \\
\Rightarrow & \left(X_{t}, U_{t}, \int_{0}^{1} g\left(X_{t}\right) d t, \sigma \int_{0}^{1}\left|X_{t}\right|^{\gamma} \log ^{m}\left|X_{t}\right| d U_{t}+A_{0} \int_{0}^{1} f^{\prime}\left(X_{t}\right) d t\right),
\end{aligned}
$$

on $D_{\mathbb{R}^{4}}[0,1]$, where $f^{\prime}(x)=|x|^{\gamma-1} \log ^{m-1}|x|(\gamma \log |x|+m) \operatorname{sign}(x)$. If A2 holds in addition, (3.5) remains valid for any $1 / \alpha<\gamma \leq 1$, any integer $m \geq 0$ and any locally integrable function $g(x)$, where $\alpha$ is given in $\boldsymbol{A} 3(i)$.

Just as in Theorem 3.2, Theorems 3.3 and 3.4 may be extended as follows to functional weak convergence results involving the index $\gamma$.

Theorem 3.5. Suppose that $\boldsymbol{A} 1$ and $\boldsymbol{A} 3$ hold, and $m \geq 0$ is an integer.

(a). If $d_{n}^{2} / n \rightarrow \infty$, on $C[A, \infty)$ with $A>1$ we have

$$
Z_{n}(\gamma):=\frac{1}{\sqrt{n}} \sum_{k=1}^{n-1}\left|x_{n k}\right|^{\gamma} \log ^{m}\left|x_{n k}\right| w_{k} \Rightarrow \int_{0}^{1}\left|X_{t}\right|^{\gamma} \log ^{m}\left|X_{t}\right| d U_{t} .
$$


(b). If $d_{n} / \sqrt{n} \rightarrow \sigma$ with $0 \leq \sigma<\infty$, on $C[A, \infty)$ with $A>1$ we have

$$
Z_{n}(\gamma):=\frac{d_{n}}{n} \sum_{k=1}^{n-1}\left|x_{n k}\right|^{\gamma} \log ^{m}\left|x_{n k}\right| w_{k} \Rightarrow \sigma \int_{0}^{1}\left|X_{t}\right|^{\gamma} \log ^{m}\left|X_{t}\right| d U_{t}+A_{0} \int_{0}^{1} f^{\prime}\left(X_{t}\right) d t
$$

where $f^{\prime}(t)$ is defined in Theorem 3.4.

If $\boldsymbol{A} 2$ holds in addition, (3.6) and (3.7) remain valid on $C[A, \infty)$ with $A>1 / \alpha$, where $\alpha$ is given in $\boldsymbol{A} 3(i)$.

Remark 3.1. The functional limit theorems for the process $Z_{n}(\gamma)$ appearing in Theorems 3.2 and 3.5 are useful in testing linearity or polynomial regression using power transformations of regressors. See, for example, Baek et al (2015) and Cho and Phillips (2018).

\section{Conclusion}

Power function regressions provide a simple way of generalizing simpler polynomial representations and offer potential for constructing general omnibus tests for specification, as shown in Cho and Phillips (2018). These characteristics extend to nonlinear cointegrating regression models with power function regressors. The present paper provides new limit theory that enables the development of an asymptotic theory of estimation in such models, allowing for both endogeneity in the regressors and for heterogeneity in the errors. Our results lead also to a simple test of linear cointegrating structures against power function alternatives, which is pivotal with a standard normal limit in the strictly exogenous regressor case. As in earlier research on nonlinear nonstationary regression models, a key element in the asymptotics is the establishment of stochastic integral limit theory that goes beyond standard martingale and semimartingale structures. The findings in the present work add to that literature and provide a broader foundation for estimation and inference in models with these characteristics. 


\section{Proofs of the main results}

This section provides proofs of the main theorems. We first prove Theorems 3.1 - 3.4, since these theorems provide technical support for the proofs of Theorems $2.1-2.4$. We start with some basic preliminaries.

Recall $x_{n,[n t]} \Rightarrow X_{t}$ on $D_{\mathbb{R}}[0,1]$ in the sense of the uniform topology and the limit Gaussian process $X(t)$ is path continuous. It follows that

$$
\limsup _{N \rightarrow \infty} \limsup _{n \rightarrow \infty}\left[\mathbb{P}\left(\max _{1 \leq k \leq n}\left|x_{n k}\right| \geq N\right)+\mathbb{P}\left(\max _{1 \leq k \leq n}\left|x_{n k}\right| \leq 1 / N\right)\right]=0 .
$$

By the tightness of $\left\{x_{n,[n t]}\right\}_{0 \leq t \leq 1}$, for any $\varepsilon>0$ and $\delta>0$, there is some $\tilde{\delta}=\tilde{\delta}(\varepsilon, \delta)>0$ such that

$$
\mathbb{P}\left(\sup _{|s-t| \leq \tilde{\delta}}\left|x_{n,[n t]}-x_{n,[n s]}\right| \geq \delta\right) \leq \varepsilon
$$

holds for all sufficiently large $n$. In terms of (5.2), for any $\delta>0$, we have

$$
\lim _{n \rightarrow \infty} \mathbb{P}\left(\max _{0 \leq j \leq n / m} \max _{j m \leq k \leq(j+1) m}\left|x_{n k}-x_{n, j m}\right| \geq \delta\right)=0,
$$

for any $m:=m_{n} \rightarrow \infty$ satisfying $n / m \rightarrow \infty$.

We next introduce a lemma, which plays a key role in the proof of the main theorems. Let $v_{k}$ be a sequence of arbitrary stochastic processes satisfying the following conditions.

A4. (i) $\sup _{k \geq 1} E\left|v_{k}\right|<\infty$;

(ii) there exist $A_{1} \in \mathbb{R}$ and $0<m:=m_{n} \rightarrow \infty$ with $\frac{n}{m} \rightarrow \infty$ so that

$$
\max _{m \leq j \leq n-m} \mathbb{E}\left|\frac{1}{m} \sum_{k=j+1}^{j+m} v_{k}-A_{1}\right|=o(1) .
$$

Lemma 5.1. Suppose $\mathbf{A} 4$ holds. For any continuous function $H(x)$ we have

$$
\frac{1}{n} \sum_{k=1}^{n} H\left(x_{n k}\right) v_{k}=\frac{A_{1}}{n} \sum_{k=1}^{n} H\left(x_{n k}\right)+o_{P}(1) .
$$

If in addition $\sup _{k \geq 1} \mathbb{E}\left|v_{k}\right|^{\alpha}<\infty$ for some $\alpha>1$ and $x_{k} / d_{k}$ has a density $p_{k}(x)$ that is uniformly bounded by a constant $K$ for all $1 \leq k \leq n$ and $x \in \mathbb{R}$, then (5.4) remains valid if $H^{\alpha /(\alpha-1)}(x)$ is a locally integrable function.

Remark 5.1. If we are only interested in the boundedness of $\sum_{k=1}^{n} H\left(x_{n k}\right) v_{k}$, condition $\mathbf{A} 4$ is not necessary. Indeed, following the proof of Lemma 5.1, it is easy to see that

$$
\sum_{k=1}^{n} H\left(x_{n k}\right) v_{k}=O_{p}(n)
$$

holds under the same conditions in Lemma 5.1 sans A4(ii). 
Proof. For any $N>0$, we assume that $H_{N}(x)=H(x) \xi_{N}(x)$ with

$$
\xi_{N}(x)= \begin{cases}1 & |x| \leq N \\ 2-|x| / N & N<|x|<2 N \\ 0 & |x| \geq 2 N\end{cases}
$$

Let $\widetilde{\lambda}_{k}=v_{k}-A_{1}, R_{n}=\frac{1}{n} \sum_{k=1}^{n} H\left(x_{n k}\right) \widetilde{\lambda}_{k}$ and $R_{1 n}=\frac{1}{n} \sum_{k=1}^{n} H_{N}\left(x_{n k}\right) \widetilde{\lambda}_{k}$. Due to (5.1), we have

$$
\mathbb{P}\left(R_{n} \neq R_{1 n}\right) \leq \mathbb{P}\left(\max _{1 \leq k \leq n}\left|x_{n k}\right|>N\right) \rightarrow 0,
$$

as $n \rightarrow \infty$ first and then $N \rightarrow \infty$. Lemma 5.1 will follow if we prove

$$
R_{1 n}=\frac{1}{n} \sum_{k=1}^{n} H_{N}\left(x_{n k}\right) \widetilde{\lambda}_{k}=o_{P}(1),
$$

for each fixed $N \geq 1$.

We first assume that $H(x)$ is continuous. In this situation, $H_{N}(x)$ is continuous with bounded support and, for any $\epsilon>0$, there exists a $\delta_{\epsilon}>0$ so that whenever $|x-y| \leq \delta_{\epsilon}$ we have

$$
\left|H_{N}(x)-H_{N}(y)\right| \leq \epsilon
$$

Write $\Omega_{\delta_{\epsilon}}=\left\{\omega: \max _{0 \leq j \leq n / m} \max _{j m \leq k \leq(j+1) m}\left|x_{n k}-x_{n, j m}\right| \leq \delta_{\epsilon}\right\}$ and $T_{n}=[n / m]-1$, where $m$ is chosen so that $m \rightarrow \infty$ and $n / m \rightarrow \infty$. By virtue of the above facts, it is readily seen that on $\Omega_{\delta_{\epsilon}}$

$$
\begin{aligned}
\left|R_{1 n}\right| \leq & \frac{1}{n} \sum_{j=0}^{T_{n}}\left|\sum_{k=j m+1}^{(j+1) m} H_{N}\left(x_{n k}\right) \widetilde{\lambda}_{k}\right|+\frac{1}{n} \sum_{k=m T_{n}+1}^{n}\left|H_{N}\left(x_{n k}\right) \widetilde{\lambda}_{k}\right| \\
\leq & \frac{1}{n} \sum_{j=0}^{T_{n}}\left|H_{N}\left(x_{n, j m}\right)\right|\left|\sum_{k=j m+1}^{(j+1) m} \tilde{\lambda}_{k}\right|+\frac{C_{N}}{n} \sum_{k=m T_{n}+1}^{n}\left|\widetilde{\lambda}_{k}\right| \\
& +\max _{0 \leq j \leq T_{n}} \max _{j m+1 \leq k \leq(j+1) m}\left|H_{N}\left(x_{n k}\right)-H_{N}\left(x_{n, j m}\right)\right| \frac{1}{n} \sum_{k=1}^{n}\left|\widetilde{\lambda}_{k}\right| \\
\leq & \frac{C_{N}}{n} \sum_{j=0}^{T_{n}}\left|\sum_{k=j m+1}^{(j+1) m} \tilde{\lambda}_{k}\right|+\frac{C_{N}}{n} \sum_{k=m T_{n}+1}^{n}\left|\widetilde{\lambda}_{k}\right|+\frac{\epsilon}{n} \sum_{k=1}^{n}\left|\tilde{\lambda}_{k}\right|,
\end{aligned}
$$

where $C_{N}$ is a constant depending only on $N$. Now, for any $\eta_{1}>0$ and $\eta_{2}>0$, it follows from (5.3) and $\mathbf{A} 4$ that, for all sufficiently large $n$,

$$
\begin{aligned}
\mathbb{P}\left(\left|R_{1 n}\right| \geq \eta_{1}\right) \leq & \mathbb{P}\left(\bar{\Omega}_{\delta_{\epsilon}}\right)+\eta_{1}^{-1} C_{N} \max _{m \leq j \leq n-m} \mathbb{E}\left|\frac{1}{m} \sum_{k=j+1}^{j+m} v_{k}-A_{1}\right| \\
& +\frac{\eta_{1}^{-1} C_{N}}{n} \sum_{k=m T_{n}+1}^{n} \mathbb{E}\left|\widetilde{\lambda}_{k}\right|+\frac{\eta_{2}}{n} \sum_{k=1}^{n} \mathbb{E}\left|\widetilde{\lambda}_{k}\right| \leq C_{1 N} \eta_{2},
\end{aligned}
$$


by taking $\epsilon=\eta_{1} \eta_{2}$, where $\bar{\Omega}_{\delta_{\epsilon}}$ denotes the complementary set of $\Omega_{\delta_{\epsilon}}$ and $C_{1 N}$ is a constant depending only on $N$. This proves (5.6) for a continuous function $H(x)$.

We next assume that $H^{\alpha /(\alpha-1)}(x)$ is locally integrable. In this situation, for any $\epsilon>0$, since both $\int_{x} H_{N}(x) d x<\infty$ and $\int_{x} H_{N}^{\alpha /(\alpha-1)}(x) d x<\infty$, there exists a continuous function $H_{N, \epsilon}(x)$ such that

$$
\int_{x}\left|H_{N}(x)-H_{N, \epsilon}(x)\right| d x \leq \epsilon \quad \text { and } \quad \int_{x}\left|H_{N}(x)-H_{N, \epsilon}(x)\right|^{\alpha /(\alpha-1)} d x \leq \epsilon .
$$

See, for instance, Lemma 5.1.2 of Stein and Shakarchi (2005). Write

$$
\begin{aligned}
R_{1 n} & =\frac{1}{n} \sum_{k=1}^{n} H_{N, \epsilon}\left(x_{n k}\right) \widetilde{\lambda}_{k}+\frac{1}{n} \sum_{k=1}^{n}\left[H_{N}\left(x_{n k}\right)-H_{N, \epsilon}\left(x_{n k}\right)\right] \widetilde{\lambda}_{k} \\
& :=S_{n 1}+S_{n 2} .
\end{aligned}
$$

For any $\epsilon>0$, using the fact shown in the first part of the proof, we have $S_{n 1}=o_{P}(1)$.

It suffices to show that $\mathbb{E}\left|S_{n 2}\right| \rightarrow 0$ as $n \rightarrow \infty$ first and then $\epsilon \rightarrow 0$. Note that, by using (5.8) and the fact that $x_{k} / d_{k}$ has a density $p_{k}(x)$ that is uniformly bounded by a constant $K$, we have

$$
\begin{aligned}
\mathbb{E}\left|H_{N}\left(x_{n k}\right)-H_{N, \epsilon}\left(x_{n k}\right)\right|^{\alpha /(\alpha-1)} & =\int\left|H_{N}\left(y / d_{n k}\right)-H_{N, \epsilon}\left(y / d_{n k}\right)\right|^{\alpha /(\alpha-1)} p_{k}(y) d y \\
& \leq K \int\left|H_{N}\left(y / d_{n k}\right)-H_{N, \epsilon}\left(y / d_{n k}\right)\right|^{\alpha /(\alpha-1)} d y \\
& \leq K d_{n k} \int\left|H_{N}(y)-H_{N, \epsilon}(y)\right|^{\alpha /(\alpha-1)} d y \\
& \leq K \epsilon d_{n k},
\end{aligned}
$$

where $d_{n k}=d_{k}^{-1} d_{n}$. It follows from Hölder's inequality and $\frac{1}{n} \sum_{k=1}^{n} d_{k}^{-1} d_{n}=O(1)$ that

$$
\begin{aligned}
\left(\mathbb{E}\left|S_{n 2}\right|\right)^{\alpha} & \leq \sup _{k \geq 1} \mathbb{E}\left|\tilde{\lambda}_{k}\right|^{\alpha}\left\{\frac{1}{n} \sum_{k=1}^{n} \mathbb{E}\left|H_{N}\left(x_{n k}\right)-H_{N, \epsilon}\left(x_{n k}\right)\right|^{\alpha /(\alpha-1)}\right\}^{\alpha-1} \\
& \leq C\left(\frac{\epsilon}{n} \sum_{k=1}^{n} d_{k}^{-1} d_{n}\right)^{\alpha-1} \rightarrow 0 .
\end{aligned}
$$

Hence $\mathbb{E}\left|S_{n 2}\right| \rightarrow 0$, as $n \rightarrow \infty$ followed by $\epsilon \rightarrow 0$, which completes the proof of Lemma 5.1.

\subsection{Proof of Theorem 3.1}

It suffices to show (3.1) when $g(x)$ and $f^{2}(x)$ are locally integrable. The first part when $g(x)$ and $f(x)$ are continuous follows from Kurtz and Protter (1991) or Hansen (1992).

The idea is similar to that of Lemma 5.1. Let $f_{N}(x)=f(x) \xi_{N}(x)$ and $g_{N}(x)=g(x) \xi_{N}(x)$. Due to the local integrability of $g(x)$ and $f^{2}(x)$, for any $\epsilon>0$, there exist continuous functions 
$g_{N, \epsilon}(x)$ and $f_{N, \epsilon}(x)$ such that $\int_{x}\left|g_{N}(x)-g_{N, \epsilon}(x)\right| d x \leq \epsilon$,

$$
\int_{x}\left|f_{N}(x)-f_{N, \epsilon}(x)\right| d x \leq \epsilon \quad \text { and } \quad \int_{x}\left|f_{N}(x)-f_{N, \epsilon}(x)\right|^{2} d x \leq \epsilon .
$$

Note that $\sup _{n} \sum_{k=1}^{n} \mathbb{E}\left(u_{n k}-u_{n, k-1}\right)^{2}=\sup _{n} n^{-1} \sum_{k=1}^{n} \mathbb{E} u_{k}^{2} \leq \sup _{k} \mathbb{E} u_{k}^{2}<\infty$. It follows from Theorem 2.1 of Hansen (1992) that, for each $N \geq 1$ and $\epsilon>0$,

$$
\begin{aligned}
& \left(x_{n,[n t]}, u_{n,[n t]}, \frac{1}{n} \sum_{k=1}^{n} g_{N, \epsilon}\left(x_{n k}\right), \frac{1}{\sqrt{n}} \sum_{k=1}^{n-1} f_{N, \epsilon}\left(x_{n k}\right) u_{k}\right) \\
\Rightarrow & \left(X_{t}, U_{t}, \int_{0}^{1} g_{N, \epsilon}\left(X_{s}\right) d s, \quad \int_{0}^{1} f_{N, \epsilon}\left(X_{s}\right) d U_{s}\right) .
\end{aligned}
$$

Note that, uniformly for $0 \leq t \leq 1$,

$$
\begin{aligned}
& \mathbb{P}\left[\left(X_{t}, U_{t}, \int_{0}^{1} g\left(X_{s}\right) d s, \int_{0}^{1} f\left(X_{s}\right) d U_{s}\right) \neq\left(X_{t}, U_{t}, \int_{0}^{1} g_{N}\left(X_{s}\right) d s, \int_{0}^{1} f_{N}\left(X_{s}\right) d U_{s}\right)\right] \\
\leq & \mathbb{P}\left(\sup _{0 \leq s \leq 1}\left|X_{s}\right|>N\right) \rightarrow 0, \\
& \mathbb{P}\left[\left(x_{n,[n t]}, u_{n,[n t]}, \frac{1}{n} \sum_{k=1}^{n} g\left(x_{n k}\right), \frac{1}{\sqrt{n}} \sum_{k=1}^{n-1} f\left(x_{n k}\right) u_{k}\right)\right. \\
& \left.\neq\left(x_{n,[n t]}, u_{n,[n t]}, \frac{1}{n} \sum_{k=1}^{n} g_{N}\left(x_{n k}\right), \frac{1}{\sqrt{n}} \sum_{k=1}^{n-1} f_{N}\left(x_{n k}\right) u_{k}\right)\right] \\
\leq & \mathbb{P}\left(\max _{1 \leq k \leq n}\left|x_{n k}\right|>N\right) \rightarrow 0,
\end{aligned}
$$

as $n \rightarrow \infty$ first and then $N \rightarrow \infty$. It is readily seen from these facts that (3.1) will hold if we prove the following: for each $N \geq 1$,

$$
\begin{aligned}
& \frac{1}{n} \sum_{k=1}^{n} g_{N, \epsilon}\left(x_{n k}\right)-\frac{1}{n} \sum_{k=1}^{n} g_{N}\left(x_{n k}\right)=o_{P}(1), \\
& \int_{0}^{1} g_{N, \epsilon}\left(X_{t}\right) d t-\int_{0}^{1} g_{N}\left(X_{t}\right) d t=o_{P}(1), \\
I_{n}:= & \frac{1}{\sqrt{n}} \sum_{k=1}^{n-1} f_{N, \epsilon}\left(x_{n k}\right) u_{k}-\frac{1}{\sqrt{n}} \sum_{k=1}^{n-1} f_{N}\left(x_{n k}\right) u_{k}=o_{P}(1), \\
I_{1 n}:= & \int_{0}^{1} f_{N, \epsilon}\left(X_{t}\right) d U_{t}-\int_{0}^{1} f_{N}\left(X_{t}\right) d U_{t}=o_{P}(1) .
\end{aligned}
$$

as $n \rightarrow \infty$ first and then $\epsilon \rightarrow 0$.

As in the proof of Lemma 5.1, by using A2 (i) and (iii), we have

$$
\begin{aligned}
\mathbb{E} I_{n}^{2} & =\frac{1}{n} \sum_{k=1}^{n-1} \mathbb{E}\left\{\left[f_{N, \epsilon}\left(x_{n k}\right)-f_{N}\left(x_{n k}\right)\right] u_{k}\right\}^{2} \\
& \leq \frac{1}{n} \sum_{k=1}^{n-1} \mathbb{E}\left(\sup _{m \geq 1} \mathbb{E}\left(u_{m}^{2} \mid \mathcal{F}_{m-1}\right)\left[f_{N, \epsilon}\left(x_{n k}\right)-f_{N}\left(x_{n k}\right)\right]^{2}\right)
\end{aligned}
$$




$$
\leq C \frac{1}{n} \sum_{k=1}^{n} d_{k}^{-1} d_{n} \int_{x}\left|f_{N}(x)-f_{N, \epsilon}(x)\right|^{2} d x \leq C_{1} \epsilon .
$$

Hence (5.12) holds. Similarly, it follows from A2 (ii) that

$$
\begin{aligned}
\mathbb{E} I_{1 n}^{2} & \leq \int_{0}^{1} \mathbb{E}\left[f_{N, \epsilon}\left(X_{t}\right)-f_{N}\left(X_{t}\right)\right]^{2} d t \\
& \leq \int_{0}^{1} \int_{y}\left[f_{N, \epsilon}(y)-f_{N}(y)\right]^{2} p_{t}(y) d y d t \\
& \leq \sup _{x} \int_{0}^{1} p_{t}(x) d t \int_{y}\left[f_{N, \epsilon}(y)-f_{N}(y)\right]^{2} d y \leq C_{2} \epsilon,
\end{aligned}
$$

yielding (5.13). The proofs of (5.10) and (5.11) are similar and this completes the proof of Theorem 3.1.

\subsection{Proof of Theorem 3.2}

To prove (3.3), let

$$
\begin{aligned}
Y_{1, n}(\gamma) & =\frac{1}{n} \sum_{k=1}^{n}\left|x_{n k}\right|^{2 \gamma} \log ^{m_{1}}\left|x_{n k}\right|, \quad Y_{2, n}(\gamma)=\frac{1}{\sqrt{n}} \sum_{k=1}^{n}\left|x_{n k}\right|^{\gamma} \log ^{m_{2}}\left|x_{n k}\right| u_{k} ; \\
\tilde{Y}_{1, n}(\gamma) & =\frac{1}{n} \sum_{k=1}^{n}\left|x_{n k}\right|^{2 \gamma} \log ^{m_{1}}\left|x_{n k}\right| I\left(x_{n k} \mid \leq N\right), \\
\tilde{Y}_{2, n}(\gamma) & =\frac{1}{\sqrt{n}} \sum_{k=1}^{n}\left|x_{n k}\right|^{\gamma} \log ^{m_{2}}\left|x_{n k}\right| I\left(\left|x_{n k}\right| \leq N\right) u_{k} .
\end{aligned}
$$

By virtue of Theorem 3.1 and Prohorov's theorem, and noting that, for every $K>a$ and $k_{0}>0$,

$$
\begin{aligned}
& \mathbb{P}\left(\sup _{\gamma_{1}, \gamma_{2} \in[a, K],\left|\gamma_{1}-\gamma_{1}\right| \leq 2^{-k_{0}}}\left|Y_{j, n}\left(\gamma_{1}\right)-Y_{j, n}\left(\gamma_{2}\right)\right| \neq \sup _{\gamma_{1}, \gamma_{2} \in[a, K],\left|\gamma_{1}-\gamma_{1}\right| \leq 2^{-k_{0}}}\left|\tilde{Y}_{j, n}\left(\gamma_{1}\right)-\tilde{Y}_{j, n}\left(\gamma_{2}\right)\right|\right) \\
\leq & \mathbb{P}\left(\max _{1 \leq k \leq n}\left|x_{n k}\right|>N\right) \rightarrow 0, \quad \text { for } j=1 \text { and } 2,
\end{aligned}
$$

as $n \rightarrow \infty$ first and then $N \rightarrow \infty$, it suffices to show the tightness of $\tilde{Y}_{1 n}(\gamma)$ and $\tilde{Y}_{2 n}(\gamma)$ on $C[a, K]$ for each $K>0$ and $N \geq 1$.

We prove the tightness of $\tilde{Y}_{1 n}(\gamma)$ and $\tilde{Y}_{2 n}(\gamma)$ on $C[a, K]$ by using Example 2.2.12 of van der Vaart and Wellner (1996). ${ }^{6}$ In particular, it suffices to show that, for any $a \leq \gamma_{1}<\gamma_{2} \leq K$

$$
\mathbb{E}\left|\widetilde{Y}_{j, n}\left(\gamma_{1}\right)-\widetilde{Y}_{j, n}\left(\gamma_{2}\right)\right|^{p} \leq C_{N}\left|\gamma_{1}-\gamma_{2}\right|^{1+r}, \quad \text { for } j=1 \text { and } 2,
$$

for some constants $p, r>0$ and $C_{N}>0$ independent of $n$.

Write $f_{N}(x)=\left(|x|^{a}+|x|^{K}\right)^{2}\left|\log ^{2+2 m_{2}}\right| x|| I(|x| \leq N)$. It is readily seen that, when $a>0$,

$$
\mathbb{E}\left(f_{N}\left(x_{n k}\right) u_{k}^{2}\right) \leq C_{N} \sup _{k} \mathbb{E} u_{k}^{2}
$$

\footnotetext{
${ }^{6}$ We thank one of the referees for suggesting the use of this approach, which has greatly shortened the original proof.
} 
and when $a>-1 / 2$,

$$
\begin{aligned}
\mathbb{E}\left(f_{N}\left(x_{n k}\right) u_{k}^{2}\right) & \leq \mathbb{E}\left[f_{N}\left(x_{n k}\right) \mathbb{E}\left(u_{k}^{2} \mid \mathcal{F}_{k-1}\right)\right] \\
& \leq C \mathbb{E} f_{N}\left(x_{n k}\right) \leq C d_{k} / d_{n} \int f_{N}(x) d x \leq C_{N} d_{k} / d_{n}
\end{aligned}
$$

under additional condition A2, where $C_{N}$ is a constant depending only on $N$. Consequently, by noting

$$
\begin{aligned}
\left.|| x\right|^{\gamma_{1}}-|x|^{\gamma_{2}} \mid & \leq\left|\gamma_{1}-\gamma_{2}\right| \max \left\{|x|^{\gamma_{1}},|x|^{\gamma_{2}}\right\}|\log (|x|)| \\
& \leq\left|\gamma_{1}-\gamma_{2}\right|\left(|x|^{a}+|x|^{K}\right)|\log (|x|)|
\end{aligned}
$$

for any $a \leq \gamma_{1}<\gamma_{2} \leq K$ and $x \neq 0$, we have

$$
\begin{aligned}
& \mathbb{E}\left(\tilde{Y}_{2, n}\left(\gamma_{1}\right)-\tilde{Y}_{2, n}\left(\gamma_{2}\right)\right)^{2} \\
= & \frac{1}{n} \sum_{k=1}^{n} \mathbb{E}\left(\left(\left|x_{n k}\right|^{\gamma_{1}}-\left|x_{n k}\right|^{\gamma_{2}}\right)^{2} \log ^{2 m_{2}}\left|x_{n k}\right| I\left(\left|x_{n k}\right| \leq N\right) u_{k}^{2}\right) \\
\leq & \left(\gamma_{1}-\gamma_{2}\right)^{2} \frac{1}{n} \sum_{k=1}^{n} \mathbb{E}\left(f_{N}\left(x_{n k}\right) u_{k}^{2}\right) \\
\leq & \left(\gamma_{1}-\gamma_{2}\right)^{2} C_{N} \frac{1}{n} \sum_{k=1}^{n}\left[d_{k}^{-1} d_{n}+\sup _{k} \mathbb{E} u_{k}^{2}\right] \leq C_{N}\left(\gamma_{1}-\gamma_{2}\right)^{2},
\end{aligned}
$$

yielding (5.14) for $j=2$, where $C_{N}>0$ is a constant independent of $n$. Similarly, by taking $r>0$ so that $a(1+r)>-1 / 2$, it follows from Hölder's inequality and (5.15) that

$$
\begin{aligned}
& \mathbb{E}\left|\widetilde{Y}_{1, n}\left(\gamma_{1}\right)-\widetilde{Y}_{1, n}\left(\gamma_{2}\right)\right|^{1+r} \\
\leq & \frac{1}{n} \sum_{k=1}^{n} \mathbb{E}\left(\left.|| x_{n k}\right|^{2 \gamma_{1}}-\left.\left|x_{n k}\right|^{2 \gamma_{2}}\right|^{1+r} \log ^{(1+r) m_{1}}\left|x_{n k}\right| I\left(\left|x_{n k}\right| \leq N\right)\right) \\
\leq & \frac{1}{n} \sum_{k=1}^{n} \mathbb{E} f_{1 N}\left(x_{n k}\right) \leq C_{N}\left|\gamma_{1}-\gamma_{2}\right|^{1+r},
\end{aligned}
$$

where $f_{1 N}(x)=\left(|x|^{a}+|x|^{K}\right)^{2(1+r)}\left|\log ^{(1+r) m_{1}}\right| x|| I(|x| \leq N)$. This proves (5.14) for $j=1$.

\subsection{Proofs of Theorems 3.3 and 3.4}

Let $f(x)=|x|^{\gamma} \log ^{m}|x|$ where $\gamma>0$ and integer $m \geq 0$, and

$$
f^{\prime}(x)=|x|^{\gamma-1} \log ^{m-1}|x|(\gamma \log |x|+m) \operatorname{sign}(x) .
$$

Obviously, $f^{\prime}(x)$ is continuous for $\gamma>1$ and $\left[f^{\prime}(x)\right]^{\alpha /(\alpha-1)}$ is locally integrable for $\gamma>1 / \alpha$. These facts will be used in the proof without further indication. To prove Theorems 3.3 and 3.4 , we write

$$
\frac{1}{\sqrt{n}} \sum_{k=1}^{n-1}\left|x_{n k}\right|^{\gamma} \log ^{m}\left|x_{n k}\right| w_{k}
$$




$$
\begin{aligned}
& =\frac{1}{\sqrt{n}} \sum_{k=1}^{n-1} f\left(x_{n k}\right) u_{k}+\frac{1}{\sqrt{n}} \sum_{k=1}^{n-1}\left[f\left(x_{n k}\right)-f\left(x_{n, k-1}\right)\right] z_{k-1}+o_{P}(1) \\
& =\frac{1}{\sqrt{n}} \sum_{k=1}^{n-1} f\left(x_{n k}\right) u_{k}+\frac{\sqrt{n}}{d_{n}} \frac{1}{n} \sum_{k=1}^{n-1} z_{k-1} \xi_{k} f^{\prime}\left(x_{n, k-1}\right)+R_{n}+o_{P}(1)
\end{aligned}
$$

where

$$
R_{n}:=\frac{1}{\sqrt{n}} \sum_{k=1}^{n-1}\left[f\left(x_{n k}\right)-f\left(x_{n, k-1}\right)-\frac{\xi_{k}}{d_{n}} f^{\prime}\left(x_{n, k-1}\right)\right] z_{k-1} .
$$

Let $v_{k}=z_{k-1} \xi_{k}$ and $\lambda_{k}=v_{k}-\mathbb{E} v_{k}$. Recalling A3, it follows that $\sup _{k \geq 1} \mathbb{E}\left(\left|v_{k}\right|^{\alpha}+\left|\lambda_{k}\right|^{\alpha}\right)<$ $\infty$, where $\alpha>1$, and, for any $0<m \rightarrow \infty$ satisfying $n / m \rightarrow \infty$ and $\widetilde{m} \rightarrow \infty$ satisfying $\widetilde{m} / \log m \rightarrow 0$

$$
\max _{m \leq j \leq n-m}\left|\frac{1}{m} \sum_{k=j+1}^{j+m} \mathbb{E} v_{k}-A_{0}\right|=o(1), \max _{m \leq j \leq n-m}\left|\frac{1}{m} \sum_{k=j+1}^{j+m} \mathbb{E}\left(\lambda_{k} \mid \mathcal{F}_{k-\widetilde{m}}\right)\right|=o_{P}(1) .
$$

Furthermore, let $\lambda_{k}(i)=\mathbb{E}\left(\lambda_{k} \mid \mathcal{F}_{k-i}\right)-\mathbb{E}\left(\lambda_{k} \mid \mathcal{F}_{k-i-1}\right)$ and

$$
\tilde{\lambda}_{k}(i)=\mathbb{E}\left(\lambda_{k} I\left(\left|\lambda_{k}\right| \leq m^{1 / 4}\right) \mid \mathcal{F}_{k-i}\right)-\mathbb{E}\left(\lambda_{k} I\left(\lambda_{k} \mid \leq m^{1 / 4}\right) \mid \mathcal{F}_{k-i-1}\right) .
$$

By noting that $\tilde{\lambda}_{k}(i)$ is a martingale difference for each $i \geq 0$ and $\sup _{k \geq 1} \mathbb{E}\left|\lambda_{k}\right|^{\alpha}<\infty$, it is readily seen that

$$
\begin{aligned}
\mathbb{E}\left|\sum_{k=j+1}^{j+m} \lambda_{k}(i)\right| & \leq\left\{\sum_{k=j+1}^{j+m} \mathbb{E}\left(\tilde{\lambda}_{k}(i)\right)^{2}\right\}^{1 / 2}+\sum_{k=j+1}^{j+m} \mathbb{E}\left|\lambda_{k}(i)-\tilde{\lambda}_{k}(i)\right| \\
& \leq C\left(m^{1 / 2+(2-\alpha) / 8}+m^{1-\alpha / 4}\right) \leq C m \log ^{-2} m,
\end{aligned}
$$

and

$$
\begin{aligned}
& \max _{m \leq j \leq n-m} \mathbb{E}\left|\frac{1}{m} \sum_{k=j+1}^{j+m}\left(\lambda_{k}-\mathbb{E}\left(\lambda_{k} \mid \mathcal{F}_{k-\widetilde{m}}\right)\right)\right| \\
\leq & \sum_{i=0}^{\widetilde{m}-1} \max _{m \leq j \leq n-m} \frac{1}{m} \mathbb{E}\left|\sum_{k=j+1}^{j+m} \lambda_{k}(i)\right| \leq C \widetilde{m} \log ^{-2} m=o(1) .
\end{aligned}
$$

Combining these estimates, it is easy to see that $v_{k}=z_{k-1} \xi_{k}$ satisfies A4 with $A_{1}=A_{0}$. As a consequence, it follows from Lemma 5.1 that

$$
\frac{1}{n} \sum_{k=1}^{n-1} z_{k-1} \xi_{k} f^{\prime}\left(x_{n, k-1}\right)=\frac{A_{0}}{n} \sum_{k=1}^{n-1} f^{\prime}\left(x_{n, k-1}\right)+o_{P}(1)
$$

for all $\gamma>1 / \alpha$. We mention that, if $1 / \alpha<\gamma \leq 1$, to prove (5.17) we need the fact that $x_{k} / d_{k}$ has a density $p_{k}(x)$ that is uniformly bounded by a constant $K$ for all $1 \leq k \leq n$ and $x \in \mathbb{R}$, which is imposed in $\mathbf{A 2}$ (i). 
Due to (5.16) and (5.17), together with Theorem 3.1 and the continuous mapping theorem, simple algebra shows that Theorem 3.3 will follow if we have

$$
R_{n}=o_{P}(1), \text { for all } \gamma>1 / \alpha
$$

where there is no bias term due to the fact that $\sqrt{n} / d_{n} \rightarrow 0$. Similarly, Theorem 3.4 will follow if we prove

$$
\frac{d_{n}}{\sqrt{n}} R_{n}=o_{P}(1), \quad \text { for all } \gamma>1 / \alpha
$$

Indeed, for Theorem 3.4, the result comes from

$$
\begin{aligned}
& \frac{d_{n}}{n} \sum_{k=1}^{n-1}\left|x_{n k}\right|^{\gamma} \log ^{m}\left|x_{n k}\right| w_{k} \\
= & \frac{d_{n}}{\sqrt{n}} \frac{1}{\sqrt{n}} \sum_{k=1}^{n-1} f\left(x_{n k}\right) u_{k}+\frac{1}{n} \sum_{k=1}^{n-1} z_{k-1} \xi_{k} f^{\prime}\left(x_{n, k-1}\right)+\frac{d_{n}}{\sqrt{n}} R_{n}+o_{P}(1),
\end{aligned}
$$

which is a minor modification of (5.16).

We next prove (5.19) under the conditions of Theorem 3.4, where we assume $d_{n}^{2} / n \rightarrow \sigma^{2}<\infty$. The proof of (5.18) is similar but simpler. To complete the proof we use the following lemma, which will be established in Appendix B.

Lemma 5.2. Suppose that $\delta>0$ is a sufficiently small constant. For any $x, y$ with $0<|x|,|y|<$ $N$ and $|x-y|<\delta$, we have that, for any $\alpha>1$,

$$
\begin{aligned}
& \left|f(x)-f(y)-(x-y) f^{\prime}(y)\right| \\
\leq & 3|x-y|\left[\left|f^{\prime}(|x|)\right| I(|x|<2 \delta)+\left|f^{\prime}(|y|)\right| I(|y|<2 \delta)\right]+C_{\delta, N}|x-y|^{\min \{\alpha, 2\}},
\end{aligned}
$$

where $C_{\delta, N}$ is a constant only depending on $\gamma, m, \alpha, \delta$ and $N$.

We return to the proof of (5.19). By Lemma 5.2, on $\Omega_{N, \delta}:=\left\{\max _{1 \leq k \leq n}\left|x_{n k}\right| \leq N\right\} \cap$ $\left\{\max _{1 \leq k \leq n}\left|x_{n k}-x_{n, k-1}\right|<\delta\right\}$, we have

$$
\frac{d_{n}}{\sqrt{n}}\left|R_{n}\right| \leq R_{1 n}+R_{2 n}+R_{3 n}
$$

where

$$
\begin{aligned}
\left|R_{1 n}\right| & \leq \frac{3}{n} \sum_{k=1}^{n-1}\left|z_{k-1} \xi_{k}\right|\left|f^{\prime}\left(x_{n k}\right)\right| I\left(\left|x_{n k}\right| \leq 2 \delta\right) \\
\left|R_{2 n}\right| & \leq \frac{3}{n} \sum_{k=1}^{n-1}\left|z_{k-1} \xi_{k}\right|\left|f^{\prime}\left(x_{n, k-1}\right)\right| I\left(\left|x_{n, k-1}\right| \leq 2 \delta\right)
\end{aligned}
$$




$$
\left|R_{3 n}\right| \leq \frac{C_{\delta, N}}{n d_{n}{ }^{\min \{\alpha-1,1\}}} \sum_{k=1}^{n-1}\left|z_{k-1}\right|\left|\xi_{k}\right|^{\min \{\alpha, 2\}} .
$$

Since $\lim _{N \rightarrow \infty} \lim _{n \rightarrow \infty} \mathbb{P}\left(\bar{\Omega}_{N, \delta}\right)=0$ by (5.1) and (5.2), where $\bar{\Omega}_{N, \delta}$ denotes the complementary set of $\Omega_{N, \delta},(5.19)$ will follow if we prove that, for any fixed $N \in \mathbb{N}$ and $\zeta>0$,

$$
\limsup _{\delta \rightarrow 0} \limsup _{n \rightarrow \infty} \mathbb{P}\left(\left|R_{i n}\right|>\zeta, \Omega_{N, \delta}\right)=0, \quad i=1,2,3 .
$$

Let $\eta>0$ be small enough so that $\gamma-2 \eta>1$ or $\gamma-2 \eta>1 / \alpha$ whenever $\gamma>1$ or $1 \geq \gamma>1 / \alpha$, respectively. For this $\eta>0$, there exists a constant $c_{0}$, which only depends on $r$ and $m$, such that $\left|f^{\prime}(x)\right| \leq c_{0}|x|^{\gamma-\eta-1}$ for all $0<x<2 \delta$. Now, recalling that $\sup _{k \geq 1} \mathbb{E}\left|z_{k-1} \xi_{k}\right|^{\alpha}<\infty$, it follows from (5.5) with $v_{k}=z_{k-1} \xi_{k}$ that, for all $\gamma>1 / \alpha$,

$$
\begin{aligned}
\left|R_{1 n}\right| & \leq \frac{3 c_{0}}{n} \sum_{k=1}^{n-1}\left|z_{k-1} \xi_{k}\right|\left|x_{n k}\right|^{\gamma-\eta-1} I\left(\left|x_{n k}\right| \leq 2 \delta\right) \\
& \leq \frac{3 c_{0}(2 \delta)^{\eta}}{n} \sum_{k=1}^{n-1}\left|z_{k-1} \xi_{k}\right|\left|x_{n k}\right|^{\gamma-2 \eta-1}=O_{p}\left(\delta^{\eta}\right),
\end{aligned}
$$

where we have used the fact that, when $1 / \alpha<\gamma \leq 1$, A2 (i) holds and $x^{(\gamma-2 \eta-1) \alpha /(\alpha-1)}$ is locally integrable due to $\gamma-2 \eta>1 / \alpha$. This implies (5.20) for $i=1$.

Similarly, (5.20) holds for $i=2$. Since $\alpha>1$ and

$$
\sup _{k} \mathbb{E}\left(\left|z_{k-1}\right|\left|\xi_{k}\right|^{\min \{\alpha, 2\}}\right) \leq \sup _{k}\left(\mathbb{E}\left[\left(1+\left|z_{k-1}\right|\right)\left|\xi_{k}\right|\right]^{\alpha}\right)^{\frac{\min \{\alpha, 2\}}{\alpha}}<\infty
$$

it is readily seen that

$$
\frac{1}{n} \sum_{k=1}^{n-1}\left|z_{k-1}\right|\left|\xi_{k}\right|^{\min \{\alpha, 2\}}=O_{P}(1)
$$

and then (5.20) holds for $i=3$ since $d_{n} \rightarrow \infty$. Combining all these results gives (5.19). The proof of Theorem 3.3 is then complete.

\subsection{Proof of Theorem 3.5}

Similar to the proof of Theorem 3.2, by virtue of Theorems 3.3 and 3.4, we only need to prove tightness, i.e., to show that, for any $\varepsilon>0, \eta>0$ and $M>A$, there exists a $k_{0}$ such that

$$
\limsup _{n \rightarrow \infty} \mathbb{P}\left[\sup _{\gamma_{1}, \gamma_{2} \in[A, M],\left|\gamma_{1}-\gamma_{2}\right| \leq 2^{-k_{0}}}\left|Z_{n}\left(\gamma_{1}\right)-Z_{n}\left(\gamma_{2}\right)\right| \geq \varepsilon\right] \leq \eta
$$

where

$$
Z_{n}(\gamma):=\frac{a_{n}}{\sqrt{n}} \sum_{k=1}^{n-1}\left|x_{n k}\right|^{\gamma} \log ^{m}\left|x_{n k}\right| w_{k}
$$


with $a_{n}=1$ in (a) and $a_{n}=d_{n} / \sqrt{n}$ in (b).

As in (5.16), we may write

$$
\begin{aligned}
& \frac{1}{\sqrt{n}} \sum_{k=1}^{n-1}\left|x_{n k}\right|^{\gamma} \log ^{m}\left|x_{n k}\right| w_{k} \\
= & \frac{1}{\sqrt{n}} \sum_{k=1}^{n-1} f_{\gamma}\left(x_{n k}\right) u_{k}+\frac{\sqrt{n}}{d_{n}} \frac{1}{n} \sum_{k=1}^{n-1} z_{k-1} \xi_{k} f_{\gamma}^{\prime}\left(x_{n, k-1}\right)+S_{1 n}(\gamma)+S_{2 n}(\gamma) \\
= & \frac{1}{\sqrt{n}} \sum_{k=1}^{n-1} f_{\gamma}\left(x_{n k}\right) u_{k}+\frac{\sqrt{n}}{d_{n}} \frac{A_{0}}{n} \sum_{k=1}^{n-1} f_{\gamma}^{\prime}\left(x_{n, k-1}\right)+S_{1 n}(\gamma)+S_{2 n}(\gamma)+S_{3 n}(\gamma),
\end{aligned}
$$

where $f_{\gamma}(x)=|x|^{\gamma} \log ^{m}|x|, f_{\gamma}^{\prime}(x)=|x|^{\gamma-1} \log ^{m-1}|x|(\gamma \log |x|+m) \operatorname{sign}(x)$,

$$
\begin{aligned}
S_{1 n}(\gamma) & :=\frac{1}{\sqrt{n}} \sum_{k=1}^{n-1}\left[f_{\gamma}\left(x_{n k}\right)-f_{\gamma}\left(x_{n, k-1}\right)-\frac{\xi_{k}}{d_{n}} f_{\gamma}^{\prime}\left(x_{n, k-1}\right)\right] z_{k-1}, \\
S_{2 n}(\gamma) & :=\frac{1}{\sqrt{n}} f_{\gamma}\left(x_{n, n-2}\right) z_{n-2}
\end{aligned}
$$

and

$$
S_{3 n}(\gamma):=\frac{\sqrt{n}}{d_{n}} \frac{1}{n} \sum_{k=1}^{n-1}\left(z_{k-1} \xi_{k}-A_{0}\right) f_{\gamma}^{\prime}\left(x_{n, k-1}\right) .
$$

Following the same arguments as in the proof of Theorem 3.2, we deduce tightness of $\frac{1}{\sqrt{n}} \sum_{k=1}^{n-1} f_{\gamma}\left(x_{n k}\right) u_{k}$ and $\frac{A_{0}}{n} \sum_{k=1}^{n-1} f_{\gamma}^{\prime}\left(x_{n, k-1}\right)$ on $C[A, \infty)$. Thus, to prove (5.21), it suffices to show that

$$
a_{n} \sup _{\gamma \in[A, M]}\left|S_{i n}(\gamma)\right|=o_{p}(1), \quad i=1,2,3 .
$$

We proceed for each value of $i$.

(a) For $i=2,(5.22)$ is obvious, following from

$$
\sup _{\gamma \in[A, M]}\left|S_{2 n}(\gamma)\right| \leq \frac{1}{\sqrt{n}}\left(\left|x_{n, n-2}\right|^{M}+\left|x_{n, n-2}\right|^{A}\right) \log ^{m}\left|x_{n, n-2}\right|\left|z_{n-2}\right|=o_{P}(1) .
$$

(b) For $i=1$, note that

$$
\sup _{\gamma \in[A, M]}\left|f_{\gamma}^{\prime}(x)\right| \leq\left(|x|^{M-1}+|x|^{A-1}\right)\left|\log ^{m-1}\right| x||(M|\log | x||+m):=g_{M}(x) .
$$

By Lemma 5.2, on $\Omega_{N, \delta}:=\left\{\max _{1 \leq k \leq n}\left|x_{n k}\right| \leq N\right\} \cap\left\{\max _{1 \leq k \leq n}\left|x_{n k}-x_{n, k-1}\right|<\delta\right\}$, we have

$$
\frac{d_{n}}{\sqrt{n}}\left|S_{1 n}(\gamma)\right| \leq R_{1 n}(\gamma)+R_{2 n}(\gamma)+R_{3 n}(\gamma)
$$

where

$$
\sup _{\gamma \in[A, M]}\left|R_{1 n}(\gamma)\right| \leq \frac{3}{n} \sum_{k=1}^{n-1}\left|z_{k-1} \xi_{k}\right| g_{M}\left(x_{n k}\right) I\left(\left|x_{n k}\right| \leq 2 \delta\right)
$$




$$
\begin{aligned}
\sup _{\gamma \in[A, M]}\left|R_{2 n}(\gamma)\right| & \leq \frac{3}{n} \sum_{k=1}^{n-1}\left|z_{k-1} \xi_{k}\right| g_{M}\left(x_{n, k-1}\right) I\left(\left|x_{n, k-1}\right| \leq 2 \delta\right), \\
\sup _{\gamma \in[A, M]}\left|R_{3 n}(\gamma)\right| & \leq \frac{C_{\delta, N}}{n d_{n}{ }^{\min \{\alpha-1,1\}}} \sum_{k=1}^{n-1}\left|z_{k-1}\right|\left|\xi_{k}\right|^{\min \{\alpha, 2\}},
\end{aligned}
$$

where $C_{\delta, N}$ is a constant only depending on $A, M, m, \alpha, \delta$ and $N$. Now, as in the proof of (5.20), we obtain the following: for any fixed $N \in \mathbb{N}$ and $\zeta>0$,

$$
\limsup _{\delta \rightarrow 0} \limsup _{n \rightarrow \infty} \mathbb{P}\left(\sup _{\gamma \in[A, M]}\left|R_{i n}(\gamma)\right|>\zeta, \Omega_{N, \delta}\right)=0, \quad i=1,2,3
$$

This, together with the fact that $\lim _{N \rightarrow \infty} \lim _{n \rightarrow \infty} \mathbb{P}\left(\bar{\Omega}_{N, \delta}\right)=0$, implies (5.22).

(c) We now prove (5.22) for $i=3$. First note that, for any $\varepsilon>0$, there exists a $\delta_{\varepsilon} \in(0, \varepsilon)$ such that $\sup _{\gamma \in[A, M]}\left|f_{\gamma}^{\prime}(x)-f_{\gamma}^{\prime}(y)\right|<\varepsilon$ holds for any $\varepsilon \leq|x|,|y| \leq N$ with $|x-y|<\delta_{\varepsilon}$. Thus, if $|x|,|y| \leq N$ and $|x-y|<\delta_{\varepsilon}<\varepsilon$, then

$$
\begin{aligned}
& \left|f_{\gamma}^{\prime}(x) I(\varepsilon \leq|x| \leq N)-f_{\gamma}^{\prime}(y) I(\varepsilon \leq|y| \leq N)\right| \\
\leq & \left|f_{\gamma}^{\prime}(x)-f_{\gamma}^{\prime}(y)\right| I(\varepsilon \leq|y| \leq N)+\left|f_{\gamma}^{\prime}(x)\right||I(\varepsilon \leq|x| \leq N)-I(\varepsilon \leq|y| \leq N)| \\
\leq & \varepsilon+2\left|f_{\gamma}^{\prime}(x)\right| I(|x|<2 \varepsilon) .
\end{aligned}
$$

Write $\tilde{\lambda}_{k}=z_{k-1} \xi_{k}-A_{0}, T_{n}=[n / l]-1$ and

$$
\tilde{\Omega}_{N, \varepsilon}=\left\{\max _{1 \leq k \leq n}\left|x_{n k}\right| \leq N\right\} \cap\left\{\max _{0 \leq j \leq n / l} \max _{j l \leq k \leq(j+1) l}\left|x_{n k}-x_{n, j l}\right| \leq \delta_{\epsilon}\right\},
$$

where $l$ is chosen so that $l \rightarrow \infty$ and $n / l \rightarrow \infty$. On $\tilde{\Omega}_{N, \varepsilon}$, it is readily seen that

$$
\begin{aligned}
& \sup _{\gamma \in[A, M]} \frac{1}{n}\left|\sum_{k=1}^{n-1}\left(z_{k-1} \xi_{k}-A_{0}\right) f_{\gamma}^{\prime}\left(x_{n, k-1}\right)\right| \\
\leq & \sup _{\gamma \in[A, M]} \frac{1}{n} \sum_{k=1}^{n-1}\left|\tilde{\lambda}_{k}\right|\left|f_{\gamma}^{\prime}\left(x_{n, k-1}\right)\right| I\left(\left|x_{n, k-1}\right|<\varepsilon\right) \\
& +\frac{1}{n} \sup _{\gamma \in[A, M]}\left|\sum_{k=1}^{n-1} \tilde{\lambda}_{k} f_{\gamma}^{\prime}\left(x_{n, k-1}\right) I\left(\varepsilon \leq\left|x_{n, k-1}\right| \leq N\right)\right| \\
=: & T_{1 n}(\varepsilon)+T_{2 n}(\varepsilon),
\end{aligned}
$$

and similar arguments to those in the proof of Lemma 5.1 [see (5.7) there] yield

$$
\begin{array}{r}
T_{2 n}(\varepsilon) \leq \frac{C_{N, \varepsilon}}{n} \sum_{j=0}^{T_{n}}\left|\sum_{k=j l+1}^{(j+1) l} \tilde{\lambda}_{k}\right|+\frac{C_{N, \varepsilon}}{n} \sum_{k=l T_{n}+1}^{n}\left|\widetilde{\lambda}_{k}\right|+\frac{\epsilon}{n} \sum_{k=1}^{n}\left|\widetilde{\lambda}_{k}\right| \\
+\frac{2}{n} \sup _{\gamma \in[A, M]} \sum_{k=1}^{n-1}\left|\tilde{\lambda}_{k}\right|\left|f_{\gamma}^{\prime}\left(x_{n, k-1}\right)\right| I\left(\left|x_{n, k-1}\right|<2 \varepsilon\right)
\end{array}
$$




$$
=T_{3 n}(\epsilon)+2 T_{1 n}(2 \epsilon), \quad \text { say }
$$

where $C_{N, \varepsilon}$ is a constant only depending on $M, m, \varepsilon$ and $N$.

Recalling (5.23), we have

$$
T_{1 n}(2 \epsilon) \leq \frac{1}{n} \sum_{k=1}^{n-1}\left|\tilde{\lambda}_{k}\right| g_{M}\left(x_{n, k-1}\right) I\left(\left|x_{n, k-1}\right|<2 \varepsilon\right)
$$

and, as in (5.24),

$$
\limsup _{\delta \rightarrow 0} \limsup _{n \rightarrow \infty} \mathbb{P}\left(T_{1 n}(2 \epsilon)>\zeta, \tilde{\Omega}_{N, \varepsilon}\right)=0,
$$

for any fixed $N \in \mathbb{N}$ and $\zeta>0$. On the other hand, recalling $\mathbf{A 3}$, we find that $v_{k}=z_{k-1} \xi_{k}$ satisfies A4 and $\sup _{k \geq 1} \mathbb{E}\left|v_{k}\right|^{\alpha}<\infty$, implying that, for any fixed $N \in \mathbb{N}$ and $\zeta>0$,

$$
\limsup _{\varepsilon \rightarrow 0} \limsup _{n \rightarrow \infty} \mathbb{P}\left(\left|T_{3 n}(\varepsilon)\right|>\zeta, \tilde{\Omega}_{N, \varepsilon}\right)=0 .
$$

Combining (5.25) to (5.28), for any fixed $N \in \mathbb{N}$ and $\zeta>0$, we have

$$
\limsup _{\varepsilon \rightarrow 0} \limsup _{n \rightarrow \infty} \mathbb{P}\left(a_{n} \sup _{\gamma \in[A, M]}\left|S_{3 n}(\gamma)\right| \geq \zeta, \tilde{\Omega}_{N, \varepsilon}\right)=0 .
$$

This implies (5.22) for $i=3$, since, for any $\epsilon>0$,

$$
\left.\mathbb{P}\left(\overline{\tilde{\Omega}}_{N, \varepsilon}\right) \leq \mathbb{P}\left(\max _{1 \leq k \leq n}\left|x_{n k}\right|>N\right\}\right)+\mathbb{P}\left(\max _{0 \leq j \leq n / l} \max _{j l \leq k \leq(j+1) l}\left|x_{n k}-x_{n, j l}\right|>\delta_{\epsilon}\right) \rightarrow 0,
$$

as $N \rightarrow \infty$ and $n \rightarrow \infty$ by (5.3), where $\overline{\tilde{\Omega}}_{N, \varepsilon}$ denotes the complementary set of $\tilde{\Omega}_{N, \varepsilon}$. The proof of Theorem 3.5 is now completed.

\subsection{Proofs of Theorems 2.1 - 2.4}

We prove Theorems $2.1-2.4$ by verifying the conditions of Theorem A.1 in Appendix A with $g_{k}(\theta)=\beta\left|x_{k}\right|^{\gamma}, \theta=(\beta, \gamma)$, and $F_{n}=\operatorname{diag}\left[\sqrt{n} d_{n}^{\gamma_{0}} / \log d_{n}, \sqrt{n} d_{n}^{\gamma_{0}}\right]$. In these derivations the results of Theorems $3.1-3.4$ are integrally involved in providing necessary technical support in the derivation.

Prior to the main derivations we give some preliminaries. Let $\dot{g}_{k}(\theta)=\frac{\partial g_{k}(\theta)}{\partial \theta}$ and $\ddot{g}_{k}(\theta)=$ $\frac{\partial^{2} g_{k}(\theta)}{\partial \theta \partial \theta^{\prime}}$, and set $H_{n}(\theta)=\sum_{k=1}^{n} \dot{g}_{k}(\theta) \dot{g}_{k}(\theta)$ and

$$
V_{n m}=\frac{1}{n} \sum_{k=1}^{n}\left|x_{n k}\right|^{2 \gamma_{0}} \log ^{m}\left|x_{n k}\right|, \quad m=0,1,2,
$$

where $x_{n k}=x_{k} / d_{n}$. By using Corollary 3.1, we have $\left(V_{n 0}, V_{n 1}, V_{n 2}\right) \rightarrow_{D}\left(V_{1}, V_{2}, V_{3}\right)$, where $V_{i}=$ $\int_{0}^{1}\left|X_{t}\right|^{2 \gamma_{0}} \log ^{i}\left|X_{t}\right| d t, i=0,1,2$ is defined as in Theorem 2.1. Since $F_{n}^{-1}=\operatorname{diag}\left[\left(\sqrt{n} d_{n}^{\gamma_{0}}\right)^{-1} \log d_{n},\left(\sqrt{n} d_{n}^{\gamma_{0}}\right)^{-1}\right]$ and

$$
\dot{g}_{k}(\theta) \dot{g}_{k}(\theta)^{\prime}=\left(\begin{array}{cc}
\left|x_{k}\right|^{2 \gamma} & \beta\left|x_{k}\right|^{2 \gamma} \log \left|x_{k}\right| \\
\beta\left|x_{k}\right|^{2 \gamma} \log \left|x_{k}\right| & \beta^{2}\left|x_{k}\right|^{2 \gamma} \log ^{2}\left|x_{k}\right|
\end{array}\right)
$$




$$
\begin{aligned}
= & \left(\begin{array}{cc}
1 & \beta \log d_{n} \\
\log d_{n} & \beta^{2} \log ^{2} d_{n}
\end{array}\right)\left|x_{k}\right|^{2 \gamma} \\
& +\left(\begin{array}{cc}
0 & \beta \\
\beta & 2 \beta^{2} \log d_{n}
\end{array}\right)\left|x_{k}\right|^{2 \gamma} \log \frac{\left|x_{k}\right|}{d_{n}}+\left(\begin{array}{cc}
0 & 0 \\
0 & \beta^{2}
\end{array}\right)\left|x_{k}\right|^{2 \gamma} \log ^{2} \frac{\left|x_{k}\right|}{d_{n}},
\end{aligned}
$$

we may write

$$
\begin{aligned}
F_{n}^{-1} H_{n}\left(\theta_{0}\right) F_{n}^{-1} & =F_{n}^{-1} \sum_{k=1}^{n} \dot{g}_{k}\left(\theta_{0}\right) \dot{g}_{k}\left(\theta_{0}\right)^{\prime} F_{n}^{-1} \\
& =\left(\begin{array}{cc}
1 & \beta_{0} \\
\beta_{0} & \beta_{0}^{2}
\end{array}\right) V_{n 0} \log ^{2} d_{n}+\left(\begin{array}{cc}
0 & \beta_{0} \\
\beta_{0} & 2 \beta_{0}^{2}
\end{array}\right) V_{n 1} \log d_{n}+\left(\begin{array}{cc}
0 & 0 \\
0 & \beta_{0}^{2}
\end{array}\right) V_{n 2} \\
& =:\left(\begin{array}{ll}
H_{11} & H_{12} \\
H_{12} & H_{22}
\end{array}\right),
\end{aligned}
$$

where

$$
\begin{aligned}
& H_{11}=V_{n 0} \log ^{2} d_{n}, \quad H_{12}=\beta_{0}\left(V_{n 0} \log ^{2} d_{n}+V_{n 1} \log d_{n}\right), \\
& H_{22}=\beta_{0}^{2}\left(V_{n 0} \log ^{2} d_{n}+V_{n 1} \log d_{n}+V_{n 2}\right) .
\end{aligned}
$$

It is easy to show that

$$
\operatorname{det}\left|F_{n}^{-1} H_{n}\left(\theta_{0}\right) F_{n}^{-1}\right|=H_{11} H_{22}-H_{12}^{2}=\beta_{0}^{2}\left(V_{n 0} V_{n 2}-V_{n 1}^{2}\right) \log ^{2} d_{n}>0 \text { a.s., }
$$

and

$$
\begin{aligned}
\left(F_{n}^{-1} H_{n}\left(\theta_{0}\right) F_{n}^{-1}\right)^{-1}=\frac{1}{H_{11} H_{22}-H_{12}}\left(\begin{array}{cc}
H_{22} & -H_{12} \\
-H_{12} & H_{11}
\end{array}\right) \\
=\frac{V_{n 0}}{\beta_{0}^{2}\left(V_{n 0} V_{n 2}-V_{n 1}^{2}\right)}\left(\begin{array}{cc}
\beta_{0}^{2} & -\beta_{0} \\
-\beta_{0} & 1
\end{array}\right)+\frac{V_{n 1}}{\beta_{0}^{2}\left(V_{n 0} V_{n 2}-V_{n 1}^{2}\right) \log d_{n}}\left(\begin{array}{cc}
2 \beta_{0}^{2} & -\beta_{0} \\
-\beta_{0} & 0
\end{array}\right) \\
\quad+\frac{V_{n 2}}{\beta_{0}^{2}\left(V_{n 0} V_{n 2}-V_{n 1}^{2}\right) \log ^{2} d_{n}}\left(\begin{array}{cc}
\beta_{0}^{2} & 0 \\
0 & 0
\end{array}\right) .
\end{aligned}
$$

Furthermore, under one of the conditions imposed in Theorems $2.1-2.4$, we have

$$
\begin{aligned}
\lambda_{\min }\left(F_{n}^{-1} H_{n}\left(\theta_{0}\right) F_{n}^{-1}\right) & =\frac{H_{11}+H_{22}-\sqrt{\left(H_{11}+H_{22}\right)^{2}-4\left(H_{11} H_{22}-H_{12}^{2}\right)}}{2} \\
& =\frac{4\left(H_{11} H_{22}-H_{12}^{2}\right)}{2\left(H_{11}+H_{22}\right)+2 \sqrt{\left(H_{11}+H_{22}\right)^{2}-4\left(H_{11} H_{22}-H_{12}^{2}\right)}} \\
& =\frac{H_{11} H_{22}-H_{12}^{2}}{H_{11}+H_{22}}+o_{p}(1) \\
& =\frac{\beta_{0}^{2}\left(V_{n 0} V_{n 2}-V_{n 1}^{2}\right)}{\left(\beta_{0}^{2}+1\right) V_{n 0}}+o_{p}(1) \\
& \rightarrow_{D} \frac{\beta_{0}^{2}\left(V_{0} V_{2}-V_{1}^{2}\right)}{\left(\beta_{0}^{2}+1\right) V_{0}}>0, \quad \text { for } \gamma_{0}>-1 / 2 .
\end{aligned}
$$

We recall that $\mathbf{A 2}$ (i) is required to establish (5.30) only for $-1 / 2<\gamma_{0} \leq 0$. 
After these preliminaries, we are now ready to prove Theorems $2.1-2.4$. For convenience, we adopt the same notation used in Theorem A.1, namely, we let $Q_{n}(\theta)=\sum_{k=1}^{n}\left(y_{k}-g_{k}(\theta)\right)^{2}$, $S_{n}(\theta)=\frac{\partial Q_{n}(\theta)}{\partial \theta}, W_{n}(\theta)=\frac{\partial^{2} Q_{n}(\theta)}{\partial \theta \partial \theta^{\prime}}, Z_{n}=F_{n}^{-1} S_{n}\left(\theta_{0}\right)$ and $Y_{n}=F_{n}^{-1} H_{n}\left(\theta_{0}\right) F_{n}^{-1}$.

Proofs of Theorems 2.1 and 2.2.

Since $\lambda_{\min }^{-1}\left(Y_{n}\right)=O_{p}(1)$ by $(5.30)$, by using Theorem A.1, Theorems 2.1 will follow if we prove that, for $\gamma_{0}>-1 / 2\left(\mathbf{A} 2\right.$ is required only for $\left.0 \geq \gamma_{0}>-1 / 2\right)$,

$$
Y_{n}^{-1} Z_{n} \rightarrow_{D}\left(\begin{array}{c}
1 \\
-1 / \beta_{0}
\end{array}\right) \frac{V_{0} \int_{0}^{1}\left|X_{t}\right|^{\gamma_{0}} \log \left|X_{t}\right| d U_{t}-V_{1} \int_{0}^{1}\left|X_{t}\right|^{\gamma_{0}} d U_{t}}{V_{0} V_{2}-V_{1}^{2}}
$$

where $V_{i}=\int_{0}^{1}\left|X_{t}\right|^{2 \gamma_{0}} \log ^{i}\left|X_{t}\right| d t, i=0,1,2$, and

$$
\sup _{\theta:\left\|F_{n}\left(\theta-\theta_{0}\right)\right\| \leq \log d_{n}}\left\|F_{n}^{-1}\left[W_{n}(\theta)-H_{n}\left(\theta_{0}\right)\right] F_{n}^{-1}\right\|=o_{P}\left(\log ^{-2} d_{n}\right) .
$$

The proof of (5.31) follows from an application of Corollary 3.1 and the continuous mapping theorem. Indeed, under model (2.1), we have

$$
S_{n}(\theta)=-\sum_{k=1}^{n} \dot{g}_{k}(\theta) u_{k}+\sum_{k=1}^{n} \dot{g}_{k}(\theta) d_{k}(\theta),
$$

where $d_{k}(\theta)=g_{k}(\theta)-g_{k}\left(\theta_{0}\right)$. Hence, some simple algebra shows that

$$
\begin{aligned}
F_{n}^{-1} S_{n}\left(\theta_{0}\right) & =-\log d_{n}\left(\begin{array}{c}
\frac{1}{\sqrt{n}} \sum_{k=1}^{n}\left|x_{n k}\right|^{\gamma_{0}} u_{k} \\
\frac{\beta_{0}}{\sqrt{n}} \sum_{k=1}^{n}\left|x_{n k}\right|^{\gamma_{0}} u_{k}+\frac{\beta_{0}}{\sqrt{n} \log d_{n}} \sum_{k=1}^{n}\left|x_{n k}\right|^{\gamma_{0}} \log \left|x_{n k}\right| u_{k}
\end{array}\right) \\
& =-\log d_{n}\left(\begin{array}{c}
1 \\
\beta_{0}
\end{array}\right) \frac{1}{\sqrt{n}} \sum_{k=1}^{n}\left|x_{n k}\right|^{\gamma_{0}} u_{k}-\left(\begin{array}{c}
0 \\
\beta_{0}
\end{array}\right) \frac{1}{\sqrt{n}} \sum_{k=1}^{n}\left|x_{n k}\right|^{\gamma_{0}} \log \left|x_{n k}\right| u_{k} .
\end{aligned}
$$

This, together with (5.29), yields that

$$
\begin{aligned}
Y_{n}^{-1} Z_{n}= & \left(F_{n}^{-1} H_{n}\left(\theta_{0}\right) F_{n}^{-1}\right)^{-1} F_{n}^{-1} S_{n}\left(\theta_{0}\right) \\
= & -\frac{V_{n 0}}{\beta_{0}^{2}\left(V_{n 0} V_{n 2}-V_{n 1}^{2}\right)}\left(\begin{array}{cc}
\beta_{0}^{2} & -\beta_{0} \\
-\beta_{0} & 1
\end{array}\right)\left(\begin{array}{c}
0 \\
\beta_{0}
\end{array}\right) \frac{1}{\sqrt{n}} \sum_{k=1}^{n}\left|x_{n k}\right|^{\gamma_{0}} \log \left|x_{n k}\right| u_{k} \\
& -\frac{V_{n 1}}{\beta_{0}^{2}\left(V_{n 0} V_{n 2}-V_{n 1}^{2}\right)}\left(\begin{array}{cc}
2 \beta_{0}^{2} & -\beta_{0} \\
-\beta_{0} & 0
\end{array}\right)\left(\begin{array}{c}
1 \\
\beta_{0}
\end{array}\right) \frac{1}{\sqrt{n}} \sum_{k=1}^{n}\left|x_{n k}\right|^{\gamma_{0}} u_{k}+O_{P}\left(\log ^{-1} d_{n}\right) \\
= & \frac{V_{n 0}}{V_{n 0} V_{n 2}-V_{n 1}^{2}}\left(\begin{array}{c}
1 \\
-1 / \beta_{0}
\end{array}\right) \frac{1}{\sqrt{n}} \sum_{k=1}^{n}\left|x_{n k}\right|^{\gamma_{0}} \log \left|x_{n k}\right| u_{k} \\
& -\frac{V_{n 1}}{V_{n 0} V_{n 2}-V_{n 1}^{2}}\left(\begin{array}{c}
1 \\
-1 / \beta_{0}
\end{array}\right) \frac{1}{\sqrt{n}} \sum_{k=1}^{n}\left|x_{n k}\right|^{\gamma_{0}} u_{k}+O_{P}\left(\log ^{-1} d_{n}\right),
\end{aligned}
$$

implying (5.31) by Corollary 3.1 and the continuous mapping theorem. 
Note that $W_{n}(\theta)=\sum_{k=1}^{n} \dot{g}_{k}(\theta) \dot{g}_{k}(\theta)^{\prime}+\sum_{k=1}^{n} \ddot{g}_{k}(\theta)\left[d_{k}(\theta)-u_{k}\right]$, where $d_{k}(\theta)=g_{k}(\theta)-g_{k}\left(\theta_{0}\right)$. The proof of (5.32) follows by verification of the following facts: for $\gamma_{0}>-1 / 2$ (A2 is required for $\left.0 \geq \gamma_{0}>-1 / 2\right)$

$$
\begin{aligned}
\sup _{\theta:\left\|F_{n}\left(\theta-\theta_{0}\right)\right\| \leq \log d_{n}}\left\|F_{n}^{-1} \sum_{k=1}^{n}\left[\dot{g}_{k}(\theta) \dot{g}_{k}(\theta)^{\prime}-\dot{g}_{k}\left(\theta_{0}\right) \dot{g}_{k}\left(\theta_{0}\right)^{\prime}\right] F_{n}^{-1}\right\| & =o_{P}\left(\log ^{-2} d_{n}\right), \\
\sup _{\theta:\left\|F_{n}\left(\theta-\theta_{0}\right)\right\| \leq \log d_{n}}\left\|F_{n}^{-1} \sum_{k=1}^{n} \ddot{g}_{k}(\theta)\left[g_{k}(\theta)-g_{k}\left(\theta_{0}\right)\right] F_{n}^{-1}\right\| & =o_{P}\left(\log ^{-2} d_{n}\right), \\
\sup _{\theta:\left\|F_{n}\left(\theta-\theta_{0}\right)\right\| \leq \log d_{n}}\left\|F_{n}^{-1} \sum_{k=1}^{n} \ddot{g}_{k}(\theta) u_{k} F_{n}^{-1}\right\| & =o_{P}\left(\log ^{-2} d_{n}\right) .
\end{aligned}
$$

Let $(A)_{i j}$ be the $(i, j)$ entry of the matrix $A$. To prove $(5.34)$, it is sufficient to prove that

$$
\sup _{\theta:\left\|F_{n}\left(\theta-\theta_{0}\right)\right\| \leq \log d_{n}}\left(F_{n}^{-1} \sum_{k=1}^{n}\left[\dot{g}_{k}(\theta) \dot{g}_{k}(\theta)^{\prime}-\dot{g}_{k}\left(\theta_{0}\right) \dot{g}_{k}\left(\theta_{0}\right)^{\prime}\right] F_{n}^{-1}\right)_{i j}=o_{P}\left(\log ^{-2} d_{n}\right)
$$

for all $i, j=1,2$. Here we only prove the case $i=j=2$ since the other cases are similar.

Let $\varepsilon>0$ be a constant satisfying that $\gamma_{0}-\varepsilon>0$ if $\gamma_{0}>0$ or $\gamma_{0}-\varepsilon>-1 / 2$ if $-1 / 2<\gamma_{0} \leq 0$. For any $\left|\gamma-\gamma_{0}\right|<\varepsilon / 3$, we have

$$
\left.\left(\log \left|x_{k}\right|\right)^{2}|| x_{k}\right|^{2 \gamma}-\left|x_{k}\right|^{2 \gamma_{0}}\left|\leq C_{\varepsilon}\right| \gamma-\gamma_{0} \mid\left(\left|x_{k}\right|^{2 \gamma_{0}+\varepsilon}+\left|x_{k}\right|^{2 \gamma_{0}-\varepsilon}\right),
$$

where $C_{\varepsilon}$ is a constant only depending on $\varepsilon$. By using Corollary 3.1,

$$
\frac{1}{n d_{n}^{2 \gamma_{0}+\varepsilon}} \sum_{k=1}^{n}\left|x_{k}\right|^{2 \gamma_{0}+\varepsilon}=O_{p}(1), \quad \frac{1}{n d_{n}^{2 \gamma_{0}-\varepsilon}} \sum_{k=1}^{n}\left|x_{k}\right|^{2 \gamma_{0}-\varepsilon}=O_{p}(1) .
$$

Hence, for sufficiently large $n$, we have

$$
\begin{aligned}
& \sup _{\theta:\left\|F_{n}\left(\theta-\theta_{0}\right)\right\| \leq \log d_{n}}\left|\left(F_{n}^{-1} \sum_{k=1}^{n}\left[\dot{g}_{k}(\theta) \dot{g}_{k}(\theta)^{\prime}-\dot{g}_{k}\left(\theta_{0}\right) \dot{g}_{k}\left(\theta_{0}\right)^{\prime}\right] F_{n}^{-1}\right)_{22}\right| \\
= & n^{-1} d_{n}^{-2 \gamma_{0}} \sup _{\theta:\left\|F_{n}\left(\theta-\theta_{0}\right)\right\| \leq \log d_{n}}\left|\sum_{k=1}^{n}\left(\log \left|x_{k}\right|\right)^{2}\left(\beta^{2}\left|x_{k}\right|^{2 \gamma}-\beta_{0}^{2}\left|x_{k}\right|^{2 \gamma_{0}}\right)\right| \\
\leq & n^{-1} d_{n}^{-2 \gamma_{0}} \sup _{\theta:\left\|F_{n}\left(\theta-\theta_{0}\right)\right\| \leq \log d_{n}} \sum_{k=1}^{n}\left(\log \left|x_{k}\right|\right)^{2}\left(\left.\beta^{2}|| x_{k}\right|^{2 \gamma}-\left|x_{k}\right|^{2 \gamma_{0}}|+| \beta^{2}-\left.\beta_{0}^{2}|| x_{k}\right|^{2 \gamma_{0}}\right) \\
= & O_{P}(1) \times n^{-3 / 2} d_{n}^{-3 \gamma_{0}} \log ^{2} d_{n}\left(\sum_{k=1}^{n}\left|x_{k}\right|^{2 \gamma_{0}-\varepsilon}+\sum_{k=1}^{n}\left|x_{k}\right|^{2 \gamma_{0}+\varepsilon}\right) \\
= & O_{p}\left(n^{-1 / 2} d_{n}^{-\gamma_{0}+\varepsilon} \log ^{2} d_{n}\right) .
\end{aligned}
$$

This proves (5.37) for $i=j=2$ by noting that $d_{n} \rightarrow \infty$ and $d_{n}^{2}=O\left(n^{\mu}\right)$ for some $0<\mu<2$. The proof of (5.35) is similar and details are omitted.

As for (5.36), by recalling

$$
\ddot{g}_{k}(\theta):=\frac{\partial^{2} g_{k}(\theta)}{\partial \theta \partial \theta^{\prime}}=\left(\begin{array}{cc}
0 & \left|x_{k}\right|^{\gamma} \log \left|x_{k}\right| \\
\left|x_{k}\right|^{\gamma} \log \left|x_{k}\right| & \beta^{2}\left|x_{k}\right|^{\gamma} \log ^{2}\left|x_{k}\right|
\end{array}\right),
$$


we only need to prove that, for $m=1,2$,

$$
\left.\frac{\log ^{2} d_{n}}{n d_{n}^{2 \gamma_{0}}} \sup _{\left|\gamma-\gamma_{0}\right| \leq n^{-1} d_{n}^{-\gamma_{0}} \log ^{2} d_{n}}\left|\sum_{k=1}^{n}\right| x_{k}\right|^{\gamma} \log ^{m}\left|x_{k}\right| u_{k} \mid=o_{P}\left(\log ^{-2} d_{n}\right) .
$$

By using Corollary 3.1, we have

$$
\left.\frac{\log ^{2} d_{n}}{n d_{n}^{2 \gamma_{0}}}\left|\sum_{k=1}^{n}\right| x_{k}\right|^{\gamma_{0}} \log ^{m}\left|x_{k}\right| u_{k} \mid=o_{P}\left(\log ^{-2} d_{n}\right), \quad m=1,2 .
$$

Hence, it is sufficient to show that

$$
\frac{\log ^{2} d_{n}}{n d_{n}^{2 \gamma_{0}}} \sup _{\left|\gamma-\gamma_{0}\right| \leq n^{-1} d_{n}^{-\gamma_{0}} \log ^{2} d_{n}}\left|\sum_{k=1}^{n}\left(\left|x_{k}\right|^{\gamma}-\left|x_{k}\right|^{\gamma_{0}}\right) \log ^{m}\right| x_{k}\left|u_{k}\right|=o_{P}\left(\log ^{-2} d_{n}\right) .
$$

Similar to the proof of (5.34), for sufficiently large $n$, it follows from (5.5) that

$$
\begin{aligned}
& \frac{\log ^{2} d_{n}}{n d_{n}^{2 \gamma_{0}}} \sup _{\left|\gamma-\gamma_{0}\right| \leq n^{-1} d_{n}^{-\gamma_{0}} \log ^{2} d_{n}}\left|\sum_{k=1}^{n}\left(\left|x_{k}\right|^{\gamma}-\left|x_{k}\right|^{\gamma_{0}}\right) \log ^{m}\right| x_{k}\left|u_{k}\right| \\
\leq & \frac{C_{\varepsilon}^{\prime} \log ^{4} d_{n}}{n^{2} d_{n}^{3 \gamma_{0}}} \sum_{k=1}^{n}\left(\left|x_{k}\right|^{\gamma_{0}+\varepsilon}+\left|x_{k}\right|^{\gamma_{0}-\varepsilon}\right)\left|u_{k}\right| \\
= & o_{P}\left(\log ^{-2} d_{n}\right),
\end{aligned}
$$

where $C_{\varepsilon}^{\prime}$ is a constant only depending on $\varepsilon$. The proofs of Theorems 2.1 and 2.2 are now complete.

Proofs of Theorems 2.3 and 2.4.

The argument is the same as that of Theorem 2.1. To illustrate, we consider an outline of the proof of Theorem 2.4. The proof of Theorem 2.3 is the same except that we replace $u_{k}$ by $w_{k}=u_{k}+z_{k-1}-z_{k}$ and Corollary 3.1 by Theorem 3.3, rather than Theorem 3.4.

First note that, following the proof of (5.36) but replacing Corollary 3.1 by Theorem 3.4, we have

$$
\sup _{\theta:\left\|F_{n}\left(\theta-\theta_{0}\right)\right\| \leq \log d_{n}}\left\|F_{n}^{-1} \sum_{k=1}^{n} \ddot{g}_{k}(\theta) w_{k} F_{n}^{-1}\right\|=o_{P}\left(\log ^{-2} d_{n}\right) .
$$

Hence, using the same argument and notation as those of Theorem 2.1, it follows that

$$
\begin{aligned}
F_{n}\left(\hat{\theta}_{n}-\theta_{0}\right)= & -Y_{n}^{-1} Z_{n}+o_{P}(1) \\
= & -\frac{V_{n 0}}{V_{n 0} V_{n 2}-V_{n 1}^{2}}\left(\begin{array}{c}
1 \\
-1 / \beta_{0}
\end{array}\right) \frac{1}{\sqrt{n}} \sum_{k=1}^{n}\left|x_{n k}\right|^{\gamma_{0}} \log \left|x_{n k}\right| w_{k} \\
& +\frac{V_{n 1}}{V_{n 0} V_{n 2}-V_{n 1}^{2}}\left(\begin{array}{c}
1 \\
-1 / \beta_{0}
\end{array}\right) \frac{1}{\sqrt{n}} \sum_{k=1}^{n}\left|x_{n k}\right|^{\gamma_{0}} w_{k}+o_{P}(1) .
\end{aligned}
$$


Now, by using Theorem 3.4 again and the continuous mapping theorem, we have

$$
\begin{aligned}
& \frac{d_{n}}{\sqrt{n}} F_{n}\left(\hat{\theta}_{n}-\theta_{0}\right)=-\frac{V_{n 0}}{V_{n 0} V_{n 2}-V_{n 1}^{2}}\left(\begin{array}{c}
1 \\
-1 / \beta_{0}
\end{array}\right) \frac{d_{n}}{n} \sum_{k=1}^{n}\left|x_{n k}\right|^{\gamma_{0}} \log \left|x_{n k}\right| w_{k} \\
&+\frac{V_{n 1}}{V_{n 0} V_{n 2}-V_{n 1}^{2}}\left(\begin{array}{c}
1 \\
-1 / \beta_{0}
\end{array}\right) \frac{d_{n}}{n} \sum_{k=1}^{n}\left|x_{n k}\right|^{\gamma_{0}} w_{k}+o_{P}(1) \\
& \rightarrow_{D}\left(\begin{array}{c}
1 \\
-1 / \beta_{0}
\end{array}\right) \frac{V_{0} W_{1}-V_{1} W_{0}}{V_{1}^{2}-V_{0} V_{2}}
\end{aligned}
$$

as required.

\subsection{Proof of Theorem 2.5}

It is readily seen that

$$
\begin{aligned}
\hat{\beta} & =\frac{\sum_{k=1}^{n} y_{k}\left[l\left(\left|x_{k}\right|\right)-n^{-1} \sum_{j=1}^{n} l\left(\left|x_{j}\right|\right)\right]}{\sum_{k=1}^{n}\left[l\left(\left|x_{k}\right|\right)-n^{-1} \sum_{j=1}^{n} l\left(\left|x_{j}\right|\right)\right]^{2}} \\
& =\beta_{0}+\frac{\sum_{k=1}^{n} u_{k}\left[l\left(\left|x_{k}\right|\right)-n^{-1} \sum_{j=1}^{n} l\left(\left|x_{j}\right|\right)\right]}{\sum_{k=1}^{n}\left[l\left(\left|x_{k}\right|\right)-n^{-1} \sum_{j=1}^{n} l\left(\left|x_{j}\right|\right)\right]^{2}} \\
\hat{\alpha} & =\frac{1}{n} \sum_{k=1}^{n} y_{k}-\frac{\hat{\beta}}{n} \sum_{k=1}^{n} l\left(\left|x_{k}\right|\right) \\
& =\alpha_{0}+\frac{1}{n} \sum_{k=1}^{n} u_{k}-\frac{\hat{\beta}-\beta_{0}}{n} \sum_{k=1}^{n} l\left(\left|x_{k}\right|\right) .
\end{aligned}
$$

Let $l_{\lambda}^{*}(x)=\frac{l(|x| \lambda)}{l(\lambda)}-1-\epsilon(\lambda) \log |x|, a_{n k}=\log \left|x_{n k}\right|-n^{-1} \sum_{j=1}^{n} \log \left(\left|x_{n j}\right|\right), \quad$ and

$$
b_{n k}=l_{d_{n}}^{*}\left(\left|x_{n k}\right|\right)-n^{-1} \sum_{j=1}^{n} l_{d_{n}}^{*}\left(\left|x_{n j}\right|\right)
$$

where $x_{n k}=x_{k} / d_{n}$. We may write

$$
\frac{1}{l^{2}\left(d_{n}\right)} \sum_{k=1}^{n}\left[l\left(\left|x_{k}\right|\right)-n^{-1} \sum_{j=1}^{n} l\left(\left|x_{j}\right|\right)\right]^{2}=\epsilon^{2}\left(d_{n}\right) \sum_{k=1}^{n} a_{n k}^{2}+\sum_{k=1}^{n} b_{n k}^{2}+2 \epsilon\left(d_{n}\right) \sum_{k=1}^{n} a_{n k} b_{n k},
$$

and

$$
\frac{1}{l\left(d_{n}\right)} \sum_{k=1}^{n} u_{k}\left[l\left(\left|x_{k}\right|\right)-n^{-1} \sum_{j=1}^{n} l\left(\left|x_{j}\right|\right)\right]=\epsilon\left(d_{n}\right) \sum_{k=1}^{n} u_{k} a_{n k}+\sum_{k=1}^{n} u_{k} b_{n k} .
$$

Taking these facts into (5.38) and (5.39), we obtain

$$
\begin{aligned}
\sqrt{n} l\left(d_{n}\right) \epsilon\left(d_{n}\right)\left(\hat{\beta}_{n}-\beta_{0}\right) & =\frac{\frac{1}{\sqrt{n}} \sum_{k=1}^{n} u_{k} a_{n k}+\frac{1}{\sqrt{n} \epsilon\left(d_{n}\right)} \sum_{k=1}^{n} u_{k} b_{n k}}{\frac{1}{n} \sum_{k=1}^{n} a_{n k}^{2}+\frac{1}{n \epsilon^{2}\left(d_{n}\right)} \sum_{k=1}^{n} b_{n k}^{2}+\frac{2}{n \epsilon\left(d_{n}\right)} \sum_{k=1}^{n} a_{n k} b_{n k}}, \\
\sqrt{n} \epsilon\left(d_{n}\right)\left(\hat{\alpha}_{n}-\alpha_{0}\right) & =\frac{\epsilon\left(d_{n}\right)}{\sqrt{n}} \sum_{k=1}^{n} u_{k}-\sqrt{n} l\left(d_{n}\right) \epsilon\left(d_{n}\right)\left(\hat{\beta}-\beta_{0}\right) \frac{1}{n l\left(d_{n}\right)} \sum_{k=1}^{n} l\left(\left|x_{k}\right|\right) .
\end{aligned}
$$


Since $\epsilon\left(d_{n}\right) \rightarrow 0$ and $\log ^{2}|x|$ is locally integrable, by using Theorem 3.1 and the continuous mapping theorem, Theorem 2.5 will follow if we prove

$$
\begin{aligned}
\frac{1}{n \epsilon^{2}\left(d_{n}\right)} \sum_{k=1}^{n} b_{n k}^{2} & =o_{P}(1), \\
\frac{1}{\sqrt{n} \epsilon\left(d_{n}\right)} \sum_{k=1}^{n} u_{k} b_{n k} & =o_{P}(1), \\
\frac{1}{n l\left(d_{n}\right)} \sum_{k=1}^{n} l\left(\left|x_{k}\right|\right) & =1+o_{P}(1) .
\end{aligned}
$$

The idea in proving (5.40)-(5.42) is quite similar to that of Theorem 3.1. We only provide an outline for (5.41). In fact, by letting

$$
b_{n k}^{*}=l_{d_{n}}^{*}\left(\left|x_{n k}\right|\right) I\left(\left|x_{n k}\right| \leq N\right)-n^{-1} \sum_{j=1}^{n} l_{d_{n}}^{*}\left(\left|x_{n j}\right|\right) I\left(\left|x_{n j}\right| \leq N\right),
$$

we have

$$
\mathbb{P}\left(\frac{1}{\sqrt{n} \epsilon\left(d_{n}\right)} \sum_{k=1}^{n} u_{k} b_{n k} \neq \frac{1}{\sqrt{n} \epsilon\left(d_{n}\right)} \sum_{k=1}^{n} u_{k} b_{n k}^{*}\right) \leq \mathbb{P}\left(\max _{1 \leq k \leq n}\left|x_{n k}\right|>N\right) \rightarrow 0,
$$

as $n \rightarrow \infty$ first and then $N \rightarrow \infty$. This result, together with the fact that due to (2.20) and A2

$$
\begin{aligned}
\frac{1}{n \epsilon^{2}\left(d_{n}\right)} \mathbb{E}\left(\sum_{k=1}^{n} u_{k} b_{n k}^{*}\right)^{2} & \leq \frac{C}{n \epsilon^{2}\left(d_{n}\right)} \sum_{k=1}^{n} \mathbb{E} b_{n k}^{* 2} \\
& \leq \frac{C}{n \epsilon^{2}\left(d_{n}\right)} \sum_{k=1}^{n} \mathbb{E}\left[l_{d_{n}}^{*}\left(\left|x_{n k}\right|\right) I\left(\left|x_{n k}\right| \leq N\right)\right]^{2} \\
& \leq \frac{C_{1}}{n \epsilon^{2}\left(d_{n}\right)} \sum_{k=1}^{n} d_{k}^{-1} d_{n}\left[\int_{|x| \leq N} l_{d_{n}}^{*}(x) d x\right]^{2}=o(1), \quad \text { as } n \rightarrow \infty
\end{aligned}
$$

for any fixed $N \geq 1$, implies (5.41). This completes the proof of Theorem 2.5.

\section{REFERENCES}

Andrews, D. W. K. and Cheng, X. (2012). Estimation and inference with weak, semi-strong, and strong identification. Econometrica, 80, 2153-2211.

Andrews, D. W. K. and Sun, Y. (2004). Adaptive local polynomial Whittle estimation of long-range dependence. Econometrica, 72, 569-614.

Baek, Y. I., Cho, H. S. and Phillips P. C. B. (2015). Testing linearity using power transforms of regressors. Journal of Econometrics, 187, 376-384.

Bae, Y. and de Jong, R. (2007). Money demand function estimation by nonlinear cointegration. Journal of Applied Econometrics, 22(4), 767-793. 
Berkes, I. and Horváth, L. (2006). Convergence of integral functionals of stochastic processes. Econometric Theory, 22, 304-322.

Billingsley, P. (1968). Convergence of Probability Measures. Wiley.

Chan, N. and Wang, Q. (2014). Uniform convergence for nonparametric estimators with nonstationary data. Econometric Theory, 30, 1110-1133.

Chan, N. and Wang, Q. (2015). Nonlinear regression with nonstationary time series, Journal of Econometrics, 185, 182-195.

Chang, Y. and Park, J. Y. (2003). Index models with integrated time series. Journal of Econometrics, 114, 73-106.

Chang, Y., Park, J. Y. and Phillips, P. C. B. (2001). Nonlinear econometric models with cointegrated and deterministically trending regressors. Econometric Journal, 4, 1-36.

Cho, J. S. and Phillips, P. C. B. (2018). Sequentially testing polynomial model hypotheses using power transforms of regressors. Journal of Applied Econometrics, 33, 141-159.

Ethier, S. N. and Kurtz, T. G. (1986). Markov Processes: Characterization and Convergence. Wiley, New York.

Gao, J. and Phillips, P. C. B. (2013). Semiparametric estimation in triangular system equations with nonstationarity. Journal of Econometrics, 176, 59-79.

Hansen, B. (1992). Convergence to stochastic integrals for dependent heterogeneous processes. Econometric Theory, 8, 489-500.

de Jong, R. (2002). Nonlinear regression with integrated regressors but without Exogeneity,. Mimeograph, Department of Economics, Michigan State University.

Kim, S. K. and Kim, I. M. (2012). Partial parametric estimation for nonstationary nonlinear regressions. Journal of Econometrics, 167, 448-457.

Kurtz, T. G. and Protter, P. (1991). Weak limit theorems for stochastic integrals and stochastic differential equations. Annals of Probability, 19, 1035-1070.

Liang, H., Phillips, P. C. B., Wang, H. and Wang, Q. (2016). Weak convergence to stochastic integrals for econometric applications. Econometric Theory, 32, 1349-1375.

Park, J. Y. and Phillips, P. C. B. (2000). Nonstationary binary choice. Econometrica, 68, 1249-1280.

Park, J. Y. and Phillips, P. C. B. (2001). Nonlinear regressions with integrated time series. Econometrica, 69, 117-161.

Peng, J. and Wang, Q. (2018). Weak convergence to stochastic integrals under primitive conditions in nonlinear econometric models. Econometric Theory, 34, 1132-1157. 
Phillips, P. C. B. (2007). Regression with slowly varying regressors and nonlinear trends. Econometric Theory, 23, 557-614.

Phillips, P. C. B. (2015). Pitfalls and Possibilities in Predictive Regression, Journal of Financial Econometrics, 13, 521-555.

Phillips, P. C. B. and Solo, V. (1992). Asymptotics for linear processes. Annals of Statistics, 20, 971-1001.

Pötscher, B. M. (2004). Nonlinear functions and convergence to Brownian motion: beyond the continuous mapping theorem. Econometric Theory, 20, 1-22.

Shi, X. and Phillips, P. C. B. (2012). Nonlinear Cointegrating regression under weak identification. Econometric Theory, 28, 509-547.

Stein, E. M. and Shakarchi, R. (2005). Real Analysis, Measure Theory, Integration and Hilbert Spaces. Princeton University Press.

van der Vaart, A. W. and Wellner, J. A. (1996). Weak Convergence and Empirical Processes: With Applications to Statistics. Springer, New York.

Wang, Q. (2014). Martingale limit theorems revisited and non-linear cointegrating regression. Econometric Theory, 30, 509-535.

Wang, Q. (2015). Limit Theorems for Nonlinear Cointegrating Regression. World Scientific.

Wang, Q., Lin, Y. X. and Gulati, C. M. (2003). Asymptotics for general fractionally integrated processes with applications to unit root tests. Econometric Theory, 19, 143-164.

Wang, Q. and Phillips, P. C. B. (2009a). Asymptotic theory for local time density estimation and nonparametric cointegrating regression. Econometric Theory, 25, 710-738.

Wang, Q. and Phillips, P. C. B. (2009b). Structural Nonparametric Cointegrating Regression. Econometrica, 25, 1901-1948.

Wang, Q. and Phillips, P. C. B.(2016). Nonparametric cointegrating regression with endogeneity and long memory. Econometric Theory, 32, 359-401.

Wooldridge, J. M. (1994). Estimation and Inference for Dependent Processes. Handbook of Econometrics, Vol. IV, ed. by Engle, R. F. and McFadden, D. L. Amsterdam: North Holland.

\section{A A general framework for nonlinear least squares estimation}

We consider the general nonlinear parametric regression model

$$
y_{k}=g_{k}(\theta)+u_{k}
$$


where $\theta \in \Theta, \Theta$ is a subset of $\mathbb{R}^{m}, g_{k}(\theta)$ is a sequence of measurable random functions on $\Theta$ and $u_{k}$ is a sequence of error variables. This section considers estimation of the unknown parameters $\theta$ in model (A.1) by using first-order conditions. The approach taken here is similar to that used in Park and Phillips $(2000,2001)$ in the development of nonlinear nonstationary regression, which in turn utilizes the framework of Wooldridge (1994).

Let $Q_{n}(\theta)=\sum_{k=1}^{n}\left[y_{k}-g_{k}(\theta)\right]^{2}, S_{n}(\theta)=(1 / 2) \partial Q_{n}(\theta) / \partial \theta, W_{n}(\theta)=(1 / 2) \partial^{2} Q_{n}(\theta) / \partial \theta \partial \theta^{\prime}$ and

$$
H_{n}(\theta)=\sum_{k=1}^{n} \dot{g}_{k}(\theta) \dot{g}_{k}(\theta)^{\prime}, \quad \text { where } \dot{g}_{k}(\theta)=\partial g_{k}(\theta) / \partial \theta \text {. }
$$

For later use, we define $\ddot{g}_{k}(\theta)=\partial^{2} g_{k}(\theta) / \partial \theta \partial \theta^{\prime}$ and assume that these quantities exist whenever they are introduced.

To construct an estimator $\hat{\theta}_{n}$ and develop asymptotics for $\hat{\theta}_{n}$, we employ the following framework, which is a generalization of Theorem 8.1 of Wooldridge (1994). Wooldridge dealt with an abstract extremum estimation problem for possibly deterministically trending and weakly dependent time series. The approach involved a smooth objective function and regularity conditions that enabled consistency and asymptotic normality for extremum estimators to be obtained within the same framework. That framework was extended to time trend power regression in Phillips (2007) and to stochastically nonstationary time series in Park and Phillips (2000, 2001), with related subsequent work in Andrews and Sun (2004), Chan and Wang (2014) and Wang and Phillips (2016).

For a sequence of matrices $F_{n}=\operatorname{diag}\left[a_{1}(n), \ldots, a_{m}(n)\right]$, we define

$$
Z_{n}=F_{n}^{-1} S_{n}\left(\theta_{0}\right), \quad Y_{n}=F_{n}^{-1} H_{n}\left(\theta_{0}\right) F_{n}^{-1}
$$

With these components we are able to state the main result.

Theorem A.1. Suppose that $\theta_{0}$ is a finite interior point of $\Theta$, and $\lambda_{\min }^{-1}\left(Y_{n}\right)=O_{p}(1)$, where $\lambda_{\min }(A)$ denotes the smallest eigenvalue of $A$, and there exists a sequence of constants $\left\{k_{n}, n \geq 1\right\}$ satisfying $k_{n} \rightarrow \infty$ and $k_{n} \max _{1 \leq j \leq m} a_{j}(n)^{-1} \rightarrow 0$ such that $Y_{n}^{-1} Z_{n}=o_{P}\left(k_{n}\right)$ and

$$
\sup _{\theta:\left\|F_{n}\left(\theta-\theta_{0}\right)\right\| \leq k_{n}}\left\|F_{n}^{-1}\left[W_{n}(\theta)-H_{n}\left(\theta_{0}\right)\right] F_{n}^{-1}\right\|=o_{P}\left(k_{n}^{-2}\right) .
$$

Then there exists a sequence of estimators $\hat{\theta}_{n}$ such that $S_{n}\left(\hat{\theta}_{n}\right)=0$ with probability that goes to one and

$$
F_{n}\left(\hat{\theta}_{n}-\theta_{0}\right)=-Y_{n}^{-1} Z_{n}+o_{P}(1)
$$


Proof. The proof follows the same arguments as that of Theorem 4.1 in Wang and Phillips (2016), see also Andrews and Sun (2004). We provide an outline here for completeness and convenience for future reference. Let $\Theta_{n}=\left\{\theta:\left\|F_{n}\left(\theta-\theta_{0}\right)\right\| \leq k_{n}\right\}$. As $k_{n}\left\|F_{n}^{-1}\right\|=o(1)$, we may take $n$ sufficiently large so that $\Theta_{n} \subset\left\{\theta:\left\|\theta-\theta_{0}\right\| \leq \delta\right\} \subset \Theta$, for some $\delta>0$. Recall that $Q_{n}(\theta)$ is twice differentiable whenever $\left\|\theta-\theta_{0}\right\| \leq \delta$. It follows by Taylor expansion that

$$
\begin{aligned}
Q_{n}(\theta)-Q_{n}\left(\theta_{0}\right)= & 2\left(\theta-\theta_{0}\right)^{\prime} S_{n}\left(\theta_{0}\right)+\left(\theta-\theta_{0}\right)^{\prime} W_{n}\left(\theta_{1}\right)\left(\theta-\theta_{0}\right) \quad\left(\text { for some } \theta_{1} \in \Theta_{n}\right) \\
= & 2\left(\theta-\theta_{0}\right)^{\prime} S_{n}\left(\theta_{0}\right)+\left(\theta-\theta_{0}\right)^{\prime} H_{n}\left(\theta_{0}\right)\left(\theta-\theta_{0}\right)+R_{n}\left(\theta, \theta_{0}\right) \\
= & {\left[F_{n}\left(\theta-\theta_{0}\right)+Y_{n}^{-1} Z_{n}\right]^{\prime} Y_{n}\left[F_{n}\left(\theta-\theta_{0}\right)+Y_{n}^{-1} Z_{n}\right] } \\
& -Z_{n}^{\prime} Y_{n}^{-1} Z_{n}+R_{n}\left(\theta, \theta_{0}\right),
\end{aligned}
$$

uniformly for $\theta \in \Theta_{n}$, where, due to (A.2),

$$
\begin{aligned}
\sup _{\theta \in \Theta_{n}}\left|R_{n}\left(\theta, \theta_{0}\right)\right| & \leq \sup _{\theta \in \Theta_{n}} \sup _{\theta_{1} \in \Theta_{n}}\left|\left(\theta-\theta_{0}\right)^{\prime}\left[W_{n}\left(\theta_{1}\right)-H_{n}\left(\theta_{0}\right)\right]\left(\theta-\theta_{0}\right)\right| \\
& \leq k_{n}^{2} \sup _{\theta_{1} \in \Theta_{n}}\left\|F_{n}^{-1}\left[W_{n}\left(\theta_{1}\right)-H_{n}\left(\theta_{n}\right)\right] F_{n}^{-1}\right\|=o_{P}(1) .
\end{aligned}
$$

Let $\widetilde{\theta}_{n}=\theta_{0}-F_{n}^{-1} Y_{n}^{-1} Z_{n}$. Using $Y_{n}^{-1} Z_{n}=o_{P}\left(k_{n}\right)$, we have

$$
\mathbb{P}\left(\widetilde{\theta}_{n} \notin \Theta_{n}\right) \leq \mathbb{P}\left(\left\|Y_{n}^{-1} Z_{n}\right\| \geq k_{n}\right) \rightarrow 0
$$

This, together with (A.4), yields

$$
Q_{n}\left(\widetilde{\theta}_{n}\right)-Q_{n}\left(\theta_{0}\right)=-Z_{n}^{\prime} Y_{n}^{-1} Z_{n}+R_{n}\left(\widetilde{\theta}_{n}, \theta_{0}\right)
$$

where $R_{n}\left(\widetilde{\theta}_{n}, \theta_{0}\right)=o_{P}(1)$. For any $\epsilon>0$ and $n \geq 1$, now let

$$
\Theta_{n}(\epsilon)=\left\{\theta \in \Theta:\left\|F_{n}\left(\theta-\theta_{0}\right)+Y_{n}^{-1} Z_{n}\right\| \leq \epsilon\right\} .
$$

Using $Y_{n}^{-1} Z_{n}=o_{P}\left(k_{n}\right)$ again, we get $\mathbb{P}\left(\Theta_{n}(\epsilon) \subset \Theta_{n}\right) \rightarrow 1$, as $n \rightarrow \infty$. Hence, for any $\theta \in \partial \Theta_{n}(\epsilon)$, where $\partial \Theta_{n}(\epsilon)$ denotes the boundary of $\Theta_{n}(\epsilon)$, it follows from (A.4) and (A.7) that

$$
Q_{n}(\theta)-Q_{n}\left(\widetilde{\theta}_{n}\right)=\nu_{n}^{\prime} Y_{n} \nu_{n}+o_{P}(1)
$$

where $\nu_{n}$ is a vector with $\left\|\nu_{n}\right\|=\epsilon>0$. Since $\nu_{n}^{\prime} Y_{n} \nu_{n} \geq \lambda_{\min }\left(Y_{n}\right)\left\|\nu_{n}\right\|^{2}=\epsilon^{2} \lambda_{\min }\left(Y_{n}\right)$, and $\widetilde{\theta}_{n} \in \Theta_{n}(\epsilon)$, equation (A.8) implies that, for each $\epsilon>0$, the event that the minimum of $Q_{n}(\theta)$ over $\Theta_{n}(\epsilon)$ is in the interior of $\Theta_{n}(\epsilon)$ has probability that goes to one as $n \rightarrow \infty$. In particular, for each $\epsilon>0$, there exists a point $\hat{\theta}_{n}(\epsilon) \in \Theta_{n}(\epsilon)$ (not necessary unique) such that $\mathbb{P}\left(\dot{Q}_{n}\left[\hat{\theta}_{n}(\epsilon)\right]=0\right) \rightarrow 1$, as $n \rightarrow \infty$. In consequence, there exists a sequence of $\hat{\theta}_{n}=\hat{\theta}_{n}\left(1 / J_{n}\right) \in \Theta_{n}\left(1 / J_{n}\right)$ where $J_{n} \rightarrow \infty$ so that $\mathbb{P}\left(\dot{Q}_{n}\left(\hat{\theta}_{n}\right)=0\right) \rightarrow 1$, as $n \rightarrow \infty$, and (A.3) holds. 


\section{B Proof of Lemma 5.2}

For $f(x)=|x|^{\gamma} \log ^{m}|x|$ and any $x, y \neq 0$, we have

$$
\left|f(x)-f(y)-(|x|-|y|) f^{\prime}(y)\right|=\frac{1}{2}(|x|-|y|)^{2}\left|f^{\prime \prime}\left(z_{0}\right)\right| \leq \frac{1}{2}(x-y)^{2}\left|f^{\prime \prime}\left(z_{0}\right)\right|,
$$

where $z_{0}$ lies between $|x|$ and $|y|$, and

$$
f^{\prime \prime}(z)=z^{\gamma-2} \log ^{m-2} z\left[\gamma(\gamma-1) \log ^{2} z+m(2 \gamma-1) \log z+m(m-1)\right], \quad z>0
$$

Hence, if $0<|x|,|y|<N$ and $|x-y| \leq \delta$, then

$$
\begin{aligned}
& \left|f(x)-f(y)-(|x|-|y|) f^{\prime}(y)\right| I(|x|>\delta,|y|>\delta) \\
\leq & \frac{1}{2} \sup _{\delta<z<N}\left|f^{\prime \prime}(z)\right|(x-y)^{2} \leq C_{\delta, N}|x-y|^{\min \{\alpha, 2\}},
\end{aligned}
$$

where $C_{\delta, N}=\frac{1}{2} \delta^{\max \{2-\alpha, 0\}} \sup _{\delta<z<N}\left|f^{\prime \prime}(z)\right|<\infty$.

For sufficiently small $\delta>0$, we have either $f^{\prime \prime}(z)>0$ for all $0<z<2 \delta$ or $f^{\prime \prime}(z)<0$ for all $0<z<2 \delta$. Thus, if $|x-y| \leq \delta$, then

$$
\begin{aligned}
& \left|f(x)-f(y)-(|x|-|y|) f^{\prime}(y)\right| I(|x|<\delta \text { or }|y|<\delta) \\
\leq & \left|(|x|-|y|)\left(f^{\prime}\left(z_{1}\right)-f^{\prime}(y)\right)\right| I(|x|<2 \delta,|y|<2 \delta) \\
\leq & |x-y|\left|f^{\prime}(x)-f^{\prime}(|y|)\right| I(|x|<2 \delta,|y|<2 \delta) \\
\leq & |x-y|\left[\left|f^{\prime}(x)\right| I(|x|<2 \delta)+\left|f^{\prime}(y)\right| I(|y|<2 \delta)\right],
\end{aligned}
$$

where $z_{1}$ lies between $|x|$ and $|y|$. Note that for any $x, y \neq 0$, we may write

$$
\begin{aligned}
(|x|-|y|) f^{\prime}(y)= & (|x|-|y|) f^{\prime}(y) I(x y>0)+(|x|-|y|) f^{\prime}(y) I(x y<0) \\
= & (x-y) \operatorname{sign}(x) f^{\prime}(y) I(x y>0)+(|x|-|y|) f^{\prime}(y) I(x y<0) \\
= & (x-y) \operatorname{sign}(x) f^{\prime}(y)-(x-y) \operatorname{sign}(x) f^{\prime}(y) I(x y<0) \\
& +(|x|-|y|) f^{\prime}(y) I(x y<0) .
\end{aligned}
$$

Hence, for any $|x-y|<\delta$,

$$
\begin{aligned}
& \left|(|x|-|y|) f^{\prime}(y)-(x-y) \operatorname{sign}(x) f^{\prime}(y)\right| \\
\leq & 2|x-y|\left|f^{\prime}(y)\right|[I(x>0, y<0)+I(x \leq 0, y>0)] \\
\leq & 2|x-y|\left|f^{\prime}(y)\right| I(|x| \leq \delta) .
\end{aligned}
$$

Lemma 5.2 now follows from (B.1)-(B.3). 